Linköping Studies in Science and Technology

Licentiate Thesis No. 1928

\title{
Epitaxial strategies for defect reduction in GaN for vertical power devices
}

Rosalía Delgado Carrascón

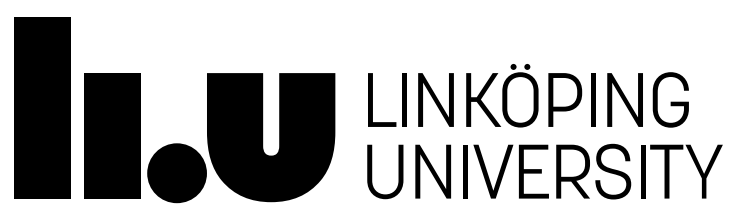





\title{
Epitaxial strategies for defect reduction in GaN for vertical power devices
}

\author{
Rosalia Delgado Carrascon
}

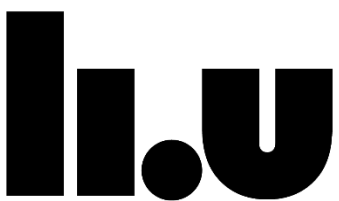

LINKÖPING UNIVERSITY

Department of Physics, Chemistry and Biology (IFM)

Linköping University, SE-581-83, Sweden

Linköping 2022 
(C) Rosalia Delgado Carrascon, 2022

Published article has been reprinted with the permission of the copyright holder.

Printed in Sweden by LiU-Tryck, Linköping, Sweden, 2022

ISBN 978-91-7929-246-1 (print)

ISBN 978-91-7929-247-8 (PDF)

https://doi.org/10.3384/9789179292478

ISSN 0280-7971 


\begin{abstract}
Group-III nitride materials, gallium nitride (GaN), aluminum nitride (AlN) and indium nitride ( $\mathrm{InN}$ ) have direct band gaps with band gap energies ranging from the infrared $(\mathrm{InN})$ to the ultraviolet $(\mathrm{GaN})$ and to the deep ultraviolet (AlN) wavelengths, covering the entire spectral range from $0.7 \mathrm{eV}$ to $6.2 \mathrm{eV}$ upon alloying. The invention of the GaN-based blue LEDs, for which the Nobel prize in Physics was awarded in 2014, has opened up avenues for exploration of III-Nitride material and device technologies, and has inspired generations of researchers in the semiconductor field. Group-III nitrides have also been demonstrated to be among the most promising semiconductors for next generation of efficient high-power, high-temperature and high-frequency electronic devices.

The need to build a sustainable and efficient energy system motivates the development of vertical GaN transistors and diodes for applications with power ratings of 50-150 kW, e.g., in electric vehicles and industrial inverters. The key is to grow $\mathrm{GaN}$ layers with low concentration of defects (impurities and dislocations), which enables an expansion in both voltage and current ratings and reduction of cost. Despite intense investigations and impressive advances in the field, defects are still a major problem which hinders exploiting the full potential of $\mathrm{GaN}$ in power electronics. This Licentiate thesis focuses on the development of two different epitaxial approaches in MOCVD for reducing dislocation densities in GaN with controlled doping for power device applications: i) growth of planar GaN layers trough NWs reformation, which can be further employed as templates for a subsequent growth of thick drift layers and ii) homoepitaxial $\mathrm{GaN}$ growth. Special attention is put on understanding homoepitaxial growth under different nucleation schemes and thermal stability of GaN. We have established conditions in homoepitaxy to deliver stateof-the-art GaN material with low impurity levels combined with a reasonable growth rate suitable for growth of thick drift layers.

The results are summarized in two papers: In Paper I we investigate GaN layers with different thicknesses on reformed GaN NW templates and highlight this approach as an alternative to the expensive GaN HVPE substrates. The sapphire used as a substrate limits to some extent the reduction of threading dislocations, however, the resulting GaN material presents smooth surfaces and thermal conductivity close to the value for bulk $\mathrm{GaN}$. Hence, this approach can be used for the development GaN as an active material for power devices on various substrates. In Paper II extensive study of homoepitaxial GaN growth by hot-wall MOCVD is presented together with results on the thermal stability of GaN under typical conditions used in our growth reactor. Understanding the evolution of GaN surface under different gas compositions and temperatures allows us to predict optimum homoepitaxial conditions. Analysis in the framework of Ga supersaturation
\end{abstract}


of epilayers simultaneously grown on GaN templates and on GaN HVPE substrates reveals that residual strain and screw dislocation densities affect $\mathrm{GaN}$ nucleation and growth and lead to distinctively different morphologies on GaN templates and native substrates, respectively. The established comprehensive picture provides guidance for designing strategies for growth conditions optimization in homoepitaxy. We demonstrate homoepitaxial GaN-on-GaN grown under optimum growth conditions with state-of-the-art smooth surface with an rms value of $0.021 \mathrm{~nm}$ and an average TDD of $1.4 \times 10^{6} \mathrm{~cm}^{-2}$ which provide good basis for augmenting power device structures.

Future work will be focused on GaN NWs reformation on different substrates, $p$ - and $n$-type doping of homoepitaxial GaN with impurity control and the fabrication of pn power diode device structures for further processing and assessment by $\mathrm{C}_{3} \mathrm{NiT}$ partners. 


\section{POPULÄRVETENSKAPLIG SAMMANFATTNING}

Grupp III-nitrider är halvledare med direkta bandgap där bandgapsenergierna spänner från det infraröda till djupt ultravioletta banden. Tillräknade i den gruppen är galliumnitrid $(\mathrm{GaN})$, aluminiumnitrid (AlN) samt indiumnitrid $(\mathrm{InN})$ som tillsammans kan realisera alla bandgapsenergier från $0.7 \mathrm{eV}(\mathrm{InN})$ till $6.2 \mathrm{eV}$ (AlN) genom legering. Utvecklingen av GaN-baserade blå LED:er, som tilldelades 2014 års Nobelpris i fysik, har öppnat många nya dörrar inom III-nitridforskning och skapat många nya tillämpningar av halvledarmaterial. Till exempel har grupp III-nitrider påvisats mycket lovande som nästa generations högeffekts- och högfrekvenskomponenter inom elektroniken. Efterfrågan på hållbara och effektiva energisystem har drivit utvecklingen av vertikala GaN-transistorer och dioder för tillämpning inom 50-150 kW omfånget, så som elektriska fordon och industriella växelriktare. Nyckeln ligger i att växa lager av GaN med låg konsentration av defekter (orenheter och dislokations), som både kan öka spänningsfönstret och strömstyrkan och samtidigt reducera kostnaden. Defekter har däremot varit svåra att kontrollera och trots mänger av framsteg är det fortfarande den stora utmaningen för att fullt kunna utnyttja potentialen av GaN inom elektronik. Den här licentiatavhandlingen fokuserar på utveckling av två olika epitaxiella framföranden genom metallorganisk kemisk ångdeposition (MOCVD) för att reducera dislokationstäthet i GaN och kontrollera doping för högeffektstillämpningar: i) plantillväxt av GaN-lager genom nanotråds-återformation, som också kan användas som grund för en utökad tillväxt av driftlager, och ii) homoepitaxiell GaN-tillväxt. Speciellt fokus har satts på att förstå homoepitaxiell tillväxt under olika kärnbildningsscenarios av GaN och dess termiska stabilitet. Licentiatsavhandlingen tar också upp vilka tillväxtstillstånd som krävs för att uppnå högkvalitet av homoepitaxiell $\mathrm{GaN}$ med låg orenhet och tillräcklig tillväxtshastiget för att växa tjocka driftlager. Resultaten är summerade in två artiklar: I Paper I undersöker vi GaN-lager med olika tjocklekar tillväxta på återformade nanotrådar av GaN och visar att det är ett alternativ till den dyrare HVPE-metoden. Däremot så begränsar safirsubstraten som används till viss del hur pass låg dislokationtäthet som kan uppnås. Substraten ger däremot upphov en jämn yta med en termiska ledningsförmåga som är nära bulkvärden. Sammantaget finns god potential för att integrera metoden för utveckling av GaN som aktivt material för högeffektskomponenter på främmande substrat. I Paper II presenteras en detaljerad studie av homoepitaxiell tillväxt av GaN genom hetväggs-MOCVD tillsammans med termisk stabilitet under olika tillväxtstilsstånd i reaktorn. Vi fokuserar på att förklara evolutionen av GaN-ytan under olika gassammansattningar och för olika temperaturer och ett optimal tillstånd för homoepitaxiell GaN tillväxt föreslås. Vi kan genom analys av 
Ga supersaturering av epilager, tillväxta på mallar av GaN och HVPE GaN substrat, visa att resterande stress och skruvdislokationstätheten påverkar kärnbildningen och tillväxten av GaN vilket leder till en rad olika ytmorfologier. Omfattande designstategier för en rad olika tillväxtstillstånd av homoepitaxiel GaN har etablerats. Homoepitaxiell GaN-på-GaN tillväxt under optimerade tillstånd demonstreras med en rekordartad ytjämnhet på RMS $0.021 \mathrm{~nm}$ och med ett medelvärde av TDD på $1.4 \times 10^{6} \mathrm{~cm}^{-2}$ vilket har lett till en god bas för förbättrade högeffektskomponenter. Framtida arbeten kommer att fokusera på GaN återformation av nanotrådar på olika substrat, $p$ - och $n$-dopad homoepitaxiell GaN ned orenhetskontroll samt tillverkning av pn-högeffektsdioder för utökade studier av $\mathrm{C}_{3} \mathrm{NiTs}$ sammarbetspartners. 


\section{PREFACE}

The licentiate thesis is written based on knowledge and research results accumulated during the graduate studies of Rosalia Delgado Carrascon at the Center for III-Nitride Technology (C3NiT-Janzen), the Department of Physics, Chemistry and Biology in the Linköping University from October 2018 to October 2021. The licentiate thesis is based on scientific papers and contains two main parts: the first part is dedicated to GaN material properties, crystal growth theory, epitaxial techniques for growth of group-III nitrides and characterization techniques which have been used in this study. The second part presents the main research results summarized in two scientific papers.

The graduate studies were accomplished under the financial supports (i) the Swedish Research Council (VR) under Grant No. 2016 - 00889, (ii) the Swedish Governmental Agency for Innovation Systems (VINNOVA) under the Competence Center Program, Grant No. 2016 - 05190, (iii) the Swedish Government Strategic Research Area in Materials Science on Functional Materials at Linköping University, Faculty Grant SFO Mat LiU No. 2009 - 00971, and (iv) the Swedish Foundation for Strategic Research (SSF), under Grant No. EM16 - 0024. 



\section{ACKNOWLEDGEMENT}

This work could not have been possible without the support and contribution of many people. I would like express my sincere gratitude to:

My main supervisor, Prof. Vanya Darakchieva for giving me the great opportunity to do my PhD studies within the Centre for III-nitride technology ( $\left.\mathrm{C}_{3} \mathrm{NiT}\right)$. Thank you for the support, encouragement and guidance.

My co-supervisor, Assoc. Prof. Plamen Paskov for always find time to provide feedback to my work.

Prof. Bo Durbeej for being my mentor during my PhD studies.

Hexagem and Lund people: Dr. Jonas Ohlsson, Dr. Jovana Colvin, Dr. Rafal Ciechonski, Dr. Mikael Björk and Prof. Lars Samuelson for their support during my studies.

Dr. Ching-Lien Hsiao. I appreciate so much his training with the CL equipment and the time he spent answering my questions.

Dr. Ildiko Farkas and Dr. Thomas Lingefelt, for being there whenever I needed them.

ALL members of $\mathrm{C}_{3} \mathrm{NiT}$ for interesting talks and discussions during the meetings. A special thanks to the PhD students within the centre who have helped and supported me in this adventure.

Prof. Fredrik Eriksson for his support with XRD, and to Dr. Steffen Richter and Dr. Nerijus Armakavicius for their timing ellipsometry results.

TEM team: Dr. Axel Persson, Dr. Ingemar Persson and Prof. Per Persson for their support with my research.

Anna Ahlgren and Louise Gustafsson for administrative support.

Prof. Tien Son Nguyen and Dr. Magnus Boman for giving me the opportunity to teach during my $\mathrm{PhD}$ studies.

Last but far from the least, thank you so much to my parents. It is very difficult to be far from you and you are always in my heart. Thank you so much to my fiancé for his endless love and support. 



\section{Contents}

$\begin{array}{ll}\text { Contents } & \text { ix }\end{array}$

1 Introduction

2 GaN and related materials 3

2.1 Crystal structure, polarity and spontaneous polarization . . . . . . . . 3

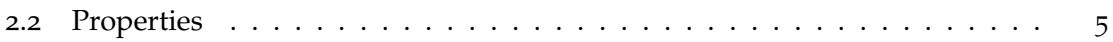

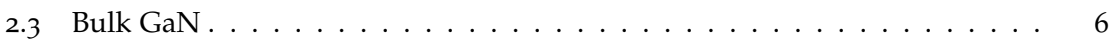

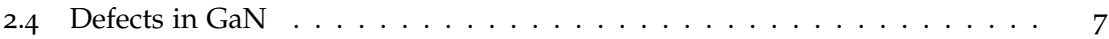

3 Crystal growth from vapor phase 9

3.1 Growth theory . . . . . . . . . . . . . . . . 9

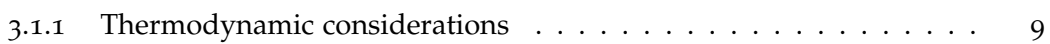

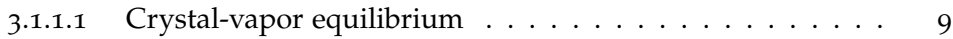

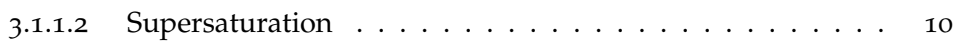

3.1.1.3 Microscopic aspects of crystal growth ......... 11

3.1 .2 Nucleation . . . . . . . . . . . . . . . . . . . . 14

3.1.3 Kinetic considerations . . . . . . . . . . . . . . . 17

3.1 .4 Equilibrium shape of crystals $\ldots \ldots \ldots \ldots \ldots \ldots \ldots$

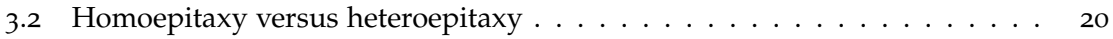

3.3 Epitaxial approaches for reduction of defects $\ldots \ldots \ldots 21$

4 Techniques for GaN growth 23

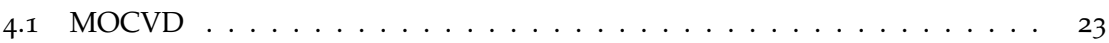

4.1.1 Hot-wall MOCVD for GaN growth . . . . . . . . . . . . 25

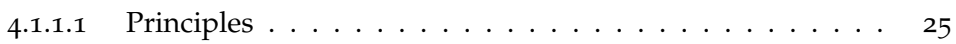

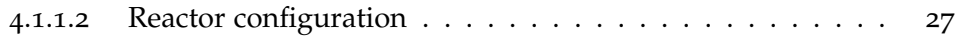

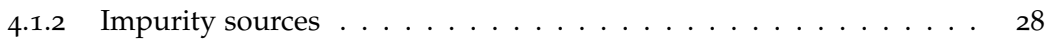

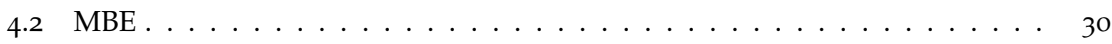




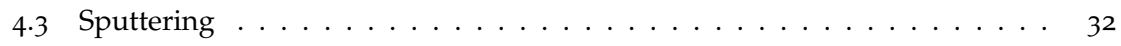

5 Characterization techniques 35

5.1 Optical microscopy . . . . . . . . . . . . . . . . . . . . . 35

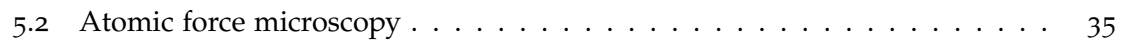

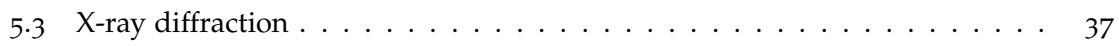

$5.3 .1 \quad$ Basic principles . . . . . . . . . . . . . . . . . 37

5.3 .2 Kinematic theory of scattering . . . . . . . . . . . . 39

5.3.3 Calculation of threading dislocation densities . . . . . . . . . 39

5.3.3.1 Screw type dislocations . . . . . . . . . . . 40

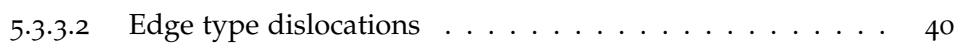

$5 \cdot 3.4$ Reciprocal space mapping . . . . . . . . . . . . . . . . $4^{1}$

5.4 Scanning electron microscopy . . . . . . . . . . . . . . $4_{41}^{1}$

5.5 Cathodoluminescence spectroscopy and imaging . . . . . . . . . . . . 43

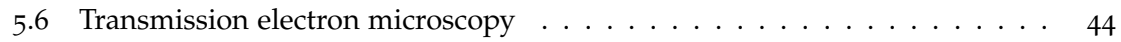

5.7 Spectroscopic ellipsometry . . . . . . . . . . . . . . . . 45

6 Summary of main results 49

6.1 Nanowires reformation . . . . . . . . . . . . . . . . . . . . . . . . . . 49

6.2 Homoepitaxial GaN growth $\ldots \ldots \ldots \ldots \ldots \ldots \ldots \ldots \ldots$

$\begin{array}{ll}\text { References } & 55\end{array}$

7 Papers $\quad 65$

7.1 Publications included in the thesis . . . . . . . . . . . . . . . 65

7.2 Publications not included in the thesis . . . . . . . . . . . . . 66 


\section{Introduction}

The invention of the GaN blue light-emitting diodes, for which Isamu Akasaki, Hiroshi Amano and Shuji Nakamura were awarded the 2014 Nobel Prize in Physics, has sparked continuous and intensive investigations of group-III nitride semiconductors and led to unprecedented advancement of the respective material technology. New growth techniques and approaches as well as different GaN nanostructures such as nanowires [1] and microprisms [2], are being explored to tune group-III nitride material properties in order to improve laser diode performance and next generation power electronic devices, for example. Wide bandgap semiconductors such as $\mathrm{GaN}$ and $\mathrm{SiC}$ have long been regarded as an alternative of Si for high-power electronic device applications [3]. Due to their high critical electric fields, $\mathrm{GaN}$ and $\mathrm{SiC}$ can enable power devices with lower capacitance and resistance for a given breakdown voltage as compared to their Si counterparts. GaN has a wider bandgap, higher critical electrical field, and higher electron saturation velocity compared to SiC. The Baliga's figure of merit (BFOM) [4] of GaN, which describes the fundamental relationship between the basic material properties and the power device performance, is more than twice larger than that of $\mathrm{SiC}$ [5]. Thus, GaN-based devices are expected to outperform SiC-based counterparts. Especially, vertical GaN-based devices promise excellent performance at high power density and high operating frequency [5, 6]. While SiC devices have achieved remarkable advances in the last decades [7], the development of $\mathrm{GaN}$ power devices has been lagging behind, mostly due to the lack of native GaN substrates. GaN-based electronic devices have been developed on different foreign substrates such as sapphire, $\mathrm{Si}$ and $\mathrm{SiC}$ [8-11]. Despite notable progress in the field, the heteroepitaxial growth does not allow to achieve high material crystal quality. The lattice and thermal expansion coefficients mismatches between GaN layers and respective foreign substrate results typically in high density of structural defects in the epilayers. Misfit dislocations generated at hetero-interfaces propagate along the entire device structures and have a detrimental effect on the device performances [12, 13]. 
Particularly, the treading dislocations (TDs) are the main reason for increased leakage current in vertical devices [14]. Therefore, in order to enable improved device performance, TD densities must be minimized. Furthermore, the use of foreign substrates limits the device design to lateral or quasi-vertical architectures and the resulting strain deteriorates reliability and yield. Different nucleation schemes, e.g., use of various buffer layers [15-17], lateral epitaxial overgrowth [18], pendeoepitaxy [19] and nanowire reformation [20] have been employed to reduce TD densities and manage strain in heterostructures.

Recently, GaN substrates fabricated by hydride vapor phase epitaxy (HVPE) and ammonothermal (ATh) growth become commercially available at a reasonable price [2123]. The typical TD density in these substrates is in the range of $10^{3}-10^{6} \mathrm{~cm}^{-2}$. Hence, epitaxial structures on GaN substrates with TD density, which are at least three orders of magnitude lower as compared to heteroepitaxially grown material, can be achieved. This enables the development of vertical GaN-based devices with outstanding performance $[5,24]$. Although GaN homoepitaxial growth on HVPE and ATh GaN substrates have been extensively studied [25-27], critical issues such as thermal decomposition of GaN [26, 28], wavy surface and formation of hillocks and ridges [29, 30], generation of interface and V-shaped dislocations [31], and impurity incorporation and segregation at the interface [27], remain to be solved. One of the main problems in homoepitaxial growth on (ooor) GaN substrates is the wavy surface morphology and the presence of macro-step terrace structures $[29,32]$. Such a morphology affects negatively the performance of vertical homoepitaxial GaN Schottky and $p-n$ diodes grown by metalorganic chemical vapor deposition (MOCVD), e. g. a high leakage current has been reported for the rough surface areas $[33,34]$. The exact origin of the wavy surface has not been clarified yet. Crystalorientation dependent diffusion and migration of adatoms, and the high supersaturation growth conditions required for homoepitaxial GaN growth are believed to play a significant role for the surface morphology [29, 34]. The formation of hillocks can be eliminated using off-cut $\left(0.25^{\circ}\right)$ substrates under growth conditions providing a large degree of adatom desorption, i.e. at high growth temperature and/or low V/III ratio [29]. However the wavy surface due to the step bunching still remained. Better understanding of the factors governing surface morphology in GaN homoepitaxy is needed in order to allow precise control and optimization of the growth conditions, and achieve GaN homoepitaxial layers with a smooth surface. 


\section{$\mathrm{GaN}$ and related materials}

\subsection{Crystal structure, polarity and spontaneous polarization}

III-V materials like GaS, InP and InSb crystalize in the zinc-blende (ZB) structure which belongs to the cubic group. In contrast, III-N materials, e.g., AlN and GaN can crystalize in $\mathrm{ZB}$ and hexagonal wurtzite (WZ), where the wurtzite structure represents the thermodynamically stable polymorph $[35,36]$. The cubic rock-salt (RS) polytype of GaN is a metastable form that can be stabilized at high pressures. The transition between WZ to RS crystal structure takes place at a pressure of $36.5 \mathrm{GPa}$ [37]. RS GaN has an indirect bang gap and different phonon spectra with respect to the $\mathrm{ZB} \mathrm{GaN} \mathrm{[38].} \mathrm{The} \mathrm{difference}$ in electronegativity between the group-III atom ( $\mathrm{Al}, \mathrm{Ga}, \mathrm{In})$ and $\mathrm{N}$ creates significant ionic contribution of the bonds which stabilizes the more compact crystal structures [39]. Depending on the relative positions of atoms in subsequent layers, different stacking sequences can be distinguished. Stacking sequence corresponding to $\mathrm{AaBbAaBbAa}$...gives rise to the hexagonal-compact lattice (hcp) structure, while atom layers stacked as AaBbC$\mathrm{cAaBbCcAa} .$. results in the face-centered cubic lattice (fcc) structure, as shown in Figure 2.1 [40]. Although the epitaxial growth of $\mathrm{ZB}-\mathrm{GaN}$ on GaAs has been demonstrated by MOVPE at relatively low growth temperatures [43], WZ GaN is the ubiquitous one since this is the thermodynamically stable polytype.

In the hexagonal system the crystal lattice is defined by two lattice constants: a and c. The perfection of the wurtzite structure can be evaluated from the value of the $c / a$ ratio with an ideal value of 1.633 [44]. The 4-axis Miller indices notation for crystal planes is normally used for WZ GaN to reflect the sixfold symmetry. In comparison to the 3-index notation for cubic system $[h k l]$, an extra index $i$ is introduced for the case of hexagonal symmetry, [hkil] with $h+k=-i$.

Due to the ionic character of the Ga-N bond, the electrons are closer to $\mathrm{N}$ than to $\mathrm{Ga}$, since $\mathrm{N}$ has a higher electronegativity. Therefore a small electron dipole forms in 
a
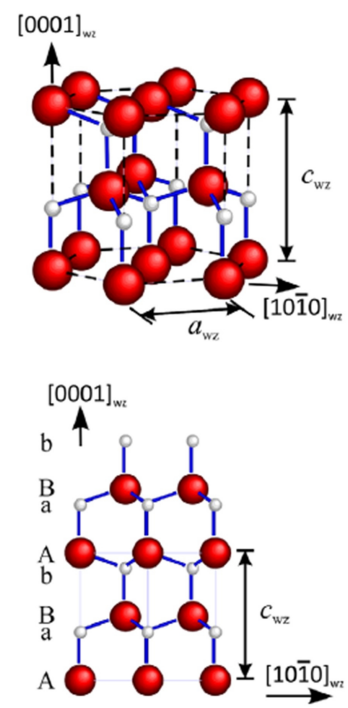

b
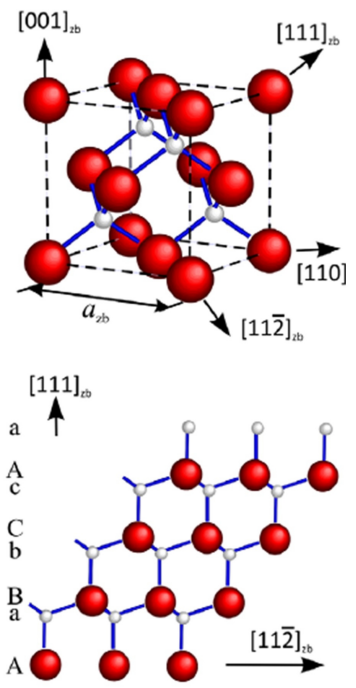

Figure 2.1: GaN common structural polytypes [41] a) Wurtzite with symmetry group $C_{6 v}^{4}\left(P 6_{3} m c\right)$ and b) Zinc-blende with symmetry group $T_{d}^{2}(F \overline{4} 3 m)$.

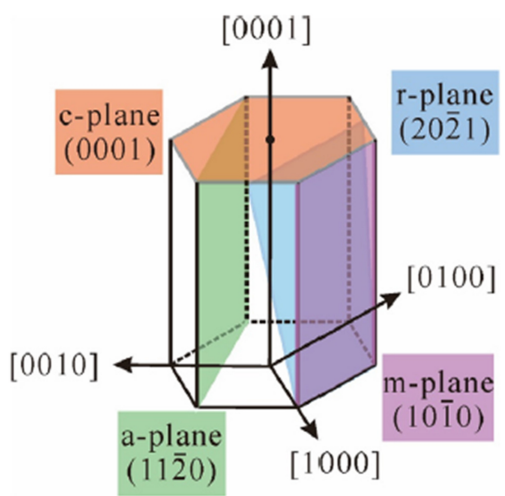

Figure 2.2: Illustration of different planes in Wurtzite III-Nitrides [42]. 
each Ga-N bond. Due to the symmetry of the hexagonal lattice, the electron dipoles are pointing out along the direction of a non-ideal tetrahedron. The dipole moments cancel out in the base of the tetrahedron but the one along the [ooo1] direction remains. Consequently, GaN exhibits spontaneous polarization along the [0001]. This property has been extensively exploited for electron confinement at e.g, the AlGaN/GaN interface, where a two dimensional electron gas (2DEG) is formed and constitutes the channel in high-electron mobility transistors (HEMTs) [45]. An important characteristic of the WZ crystal structure is the lack of inversion symmetry along the c-axis [ooo1]. This leads to two different polarities depending on the direction of the three bonds of the metal-elements with respect to the N-atom. When the group-III atom is on top of the $\mathrm{N}$ atom along [oool] i.e. the direction of the three bonds of the metal-elements are towards the substrate, the polarity is referred to as metal-polar or $+c$ polarity. On the other hand, when the three bonds of the metal-elements point upward with respect to the substrate, i.e. the group-III atom is on top of the $\mathrm{N}$ atom, the polarity is defined as N-polar or -c polarity. The N-polar face has higher absolute surface free energy than the Ga-polar face [46], which leads to differences in thermodynamic and kinetic aspects of growth. The growth of N-polar GaN tends to be accompanied by hillock formation, since the higher energetic surface leads to a slower growth kinetics [47].

Besides polar planes, there are planes where the spontaneous polarization is zero or

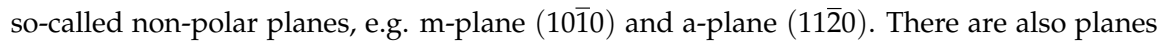
where the spontaneous polarization is between the value along the [0oo1] and 0 , termed semi-polar planes such as r-plane (2021), for example. Some of the main planes in WZ $\mathrm{GaN}$ are schematically shown in Figure 2.2. The properties of GaN grown with c-plane and with different non-polar and semi-polar planes have been widely explored [48-50]. There are less number of bonds per area in $+\mathrm{c}$ and $-\mathrm{c}$ planes than for the aforementioned semi-polar and non-polar planes, therefore their absolute surface energies are lower.

\subsection{Properties}

The physical properties of the major power semiconductors: $\mathrm{Si}, 4 \mathrm{H}-\mathrm{SiC}, \mathrm{GaN}$ and $\mathrm{AlN}$ at $300 \mathrm{~K}$ are summarized in Table 2.1. GaN, AlN and their alloys have wide and direct band-gaps, high thermal conductivity, chemical inertness that are superior to those of $\mathrm{Si}$. Consequently, III-Nitrides can enable application in high power electronic devices that can be operated at elevated temperatures. Their chemical stability, high breakdown electric field, high electron mobility and low intrinsic carrier concentrations leads to reduced leakage currents in rf-electronic devices.

The Balliga figure of merit (FOM) of AlN is the highest among the current power 
Table 2.1: Physical properties of major power semiconductors: $\mathrm{Si}, 4 \mathrm{H}-\mathrm{SiC}, \mathrm{GaN}$ and $\mathrm{AlN}$ at temperature of $300 \mathrm{~K}$.

\begin{tabular}{|c|c|c|c|c|}
\hline & Si & ${ }_{4} \mathrm{H}-\mathrm{SiC}[51,52]$ & $\operatorname{GaN}[51,53,54]$ & AlN [52] \\
\hline Band-gap energy [eV] & 1.1 & 3.2 & 3.44 & 6.2 \\
\hline Critical field [MV/cm] & 0.3 & 2.5 & $3 \cdot 4$ & 12 \\
\hline electron mobility $\left[\mathrm{cm}^{2} /\right.$ V.s] & 1300 & 800 & 1200 & 300 \\
\hline a $[\AA]$ & $5 \cdot 43$ & 3.07 & 3.189 & 3.11 \\
\hline$c[\AA]$ & & 10.08 & 5.185 & 4.979 \\
\hline $\begin{array}{l}\text { Thermal } \\
{[\mathrm{W} / \mathrm{cm} . \mathrm{K}]}\end{array}$ & 1.3 & 4.9 & $2.3-2.7$ & $2 \cdot 7-3 \cdot 7$ \\
\hline Baliga FOM & 1 & 340 & 1450 & 10067 \\
\hline
\end{tabular}

semiconductors, however, difficulties in doping currently limits its application of AlN in high power devices. In addition, the growth of AlN is challenging since the Al-N bond has a strong ionic component which causes reduced surface mobility and hence very high growth temperatures are needed. On the other hand, $\mathrm{SiC}$ is one of the most widely employed semiconductor for high power electronics and have successfully been adopted in a myriad of commercial applications. However, its reduced mobility of 400 $\mathrm{cm}^{2} V^{-1} \mathrm{~s}^{-1}$ compared to $1000 \mathrm{~cm}^{2} V^{-1} \mathrm{~S}^{-1}$ for GaN and the bipolar degradation of SiC in bipolar devices [55], has contributed to an increased interest in exploiting $\mathrm{GaN}$ for power electronics.

\subsection{Bulk GaN}

The growth of GaN single crystals by conventional methods like Czochralski and Birgman, typically employed for the growth of e.g., single crystalline $\mathrm{Si}$, is not possible due to the extremely high decomposition pressure at the melting point of GaN (Table 2.2). Although other growth methods such as flux-assisted methods [56] and growth from melt [57] have been explored, the size of the GaN single crystals is still much smaller than the required wafer dimension which limits their wide adoption. Nowadays, the most widespread techniques to fabricate GaN substrates are the ammonothermal method [58] and the halide vapor phase epitaxy (HVPE) [21-23]. The ammonothermal growth is similar to the hydrothermal growth but makes use of a solution of supercritical ammonia instead of water [59]. The growth takes place in a high-pressure autoclave at process pressure of about 1-3 kbar. The autoclave is divided into dissolution and crystallization zones. In the former, the feed-stock is dissolved. Then, by applying a gradient of temperature there is a mass transport by convection from the dissolution zone to the crystallization zone, where the initial solution is supersaturated and GaN crystallizes on native seeds. 
Table 2.2: Melting point (T) and decomposition pressure at the melting point (P) for some $\mathrm{Ga}-\mathrm{V}$ binary compounds.

\begin{tabular}{lcc}
\hline Material & $\mathrm{T}\left[{ }^{\circ} \mathrm{C}\right]$ & $\mathrm{P}[\mathrm{atm}]$ \\
\hline GaN & 2500 & 45000 \\
$\mathrm{GaP}$ & 1465 & 30 \\
$\mathrm{GaAs}$ & 1250 & 15 \\
\hline
\end{tabular}

The HVPE technique is actually not a bulk growth method however, the high growth rates, which are superior than the ammonothermal method, the low impurity incorporation and the low threading dislocations in the resulting GaN material, make this technique very promising to provide pseudo-bulk GaN substrates with thickness of several hundreds of microns. The HVPE method is based on the reaction of liquid $\mathrm{Ga}$ with $\mathrm{HCl}$ which results in the formation of $\mathrm{GaCl}_{3}$. This compound reacts with ammonia to form $\mathrm{GaN}$ in the vicinity of the substrate, typically sapphire. Strain generated in the initial stages of growth due to the high lattice mismatch between the layer and the substrate is one of the the main challenges in the fabrication of large GaN substrates by HVPE. To alleviate the strain effects epitaxial lateral overgrowth (ELO) is used in the initial growth stage. The ELO technique makes use of a mask, so the voids formed between GaN nanocolumns contribute to strain release [6o, 61]. The GaN quasi-substrates are fabricated by separation of the thick HVPE GaN from the substrate and subsequent slicing and polishing. In order to improve further the quality of the HVPE-GaN, GaN bulk substrates obtained from the ammonothermal technique were proposed as an alternative substrate [59]. However, this is related to significant increase of the HVPE GaN substrate cost.

\subsection{Defects in GaN}

No perfect solid exists, even at temperature of $0 \mathrm{~K}$. The surface itself is a type of defect due to the presence of dangling bonds. All kind of defects imply a deviation from the theoretical-predicted perfect crystal lattice. To our interest, only defects of single crystal solids, in particular GaN, are briefly summarized in Table 2.3. Dislocations have a direct deteriorating impact on GaN electric and optical properties. Commonly, they arise from lattice misfit during heteroepitaxial growth. Dislocations represent a relative displacement of two parallel planes of atoms which creates a shear stress field determined by the material shear modulus $G$ [62]. The plane containing the direction of the displacement is known as a slip plane. Dislocations are irreversible and thus represent a plastic deformation of the crystal. In this thesis we consider edge type of dislocations and screw type of dislocations. Edge dislocations are formed when an additional plane of atoms is introduced into the upper half part of the lattice being the boundary between distorted and non-distorted 
Table 2.3: Common defects found in syngle crystalline solids.

\begin{tabular}{lcc}
\hline Dimensions & Type of defect & Examples \\
\hline oD & Point & Vacancies, impurities \\
1D & Linear & Dislocations \\
2D & Planar & Stacking faults, surfaces \\
3D & Volume & Inclusions, voids, cracks \\
\hline
\end{tabular}

regions directed normal to the slip plane. Screw dislocations are generated after the slip plane has been displaced parallel to the dislocation line. This generates a helicoidal atom arrangement along the dislocation line. The nature of the dislocations are distinguished by the direction of their Burguers vector, $\mathbf{b}$, and its magnitude, which has been found to be proportional to the elastic energy of the dislocation [63]. Dislocations are unstable regions within the material since their cores are surrounded by dangling bonds which can bind electrons and thus limit the conductivity of $n$-GaN. In addition, impurity atoms may segregate along dislocations, which may interfere with material transport properties. 


\section{Crystal growth from vapor phase}

\subsection{Growth theory}

\subsubsection{Thermodynamic considerations}

\subsubsection{Crystal-vapor equilibrium}

Vapor phase and crystal, which is the substrate on which epitaxial growth occurs, are in equilibrium when the chemical potentials are equal in both phases: vapor and solid, at constant total pressure and temperature during the growth process:

$$
\mu_{V}(P, T)=\mu_{S}(P, T)
$$

The chemical potential is defined as the variation of the Gibbs free energy with respect to the number of particles:

$$
\mu=\left(\frac{\partial G}{\partial n}\right)_{P, T}
$$

As an approximation, we consider the chemical potentials of a single component, in our case $\mathrm{Ga}$, and the temperature and pressures of both phases to be equal, therefore the boundary region between both phases is flat. If the chemical potentials of both phases undertake an infinitesimal variation so that the equilibrium conditions are maintained:

$$
d \mu_{V}(P, T)=d \mu_{S}(P, T)
$$

Taking into consideration the definition of the Gibbs free energy:

$$
d G=V d P-S d T
$$

one arrives to the equation of Clapeyron, which describes the transfer of energy which 
makes possible the transition from vapor to solid required for epitaxial growth:

$$
\frac{d P}{d T}=\frac{\Delta S}{\Delta V}=\frac{\Delta h}{T \Delta V},
$$

where $\Delta S, \Delta h$ and $\Delta V$ are the variations in molar entropy, molar enthalpy and molar volume, respectively.

To describe the crystal-vapor equilibrium, we can neglect the molar volume of the crystal with respect of the vapor and consider the vapor as an ideal gas. If the enthalpy of sublimation, $\Delta h_{s u b}$, is independent of temperature, and integrating between an equilibrium state and a final state, one reaches the equation:

$$
\frac{P}{P_{0}}=\exp \left[\frac{-\Delta h_{\text {sub }}}{R}\left(\frac{1}{T}-\frac{1}{T_{0}}\right)\right],
$$

where $P_{0}$ is the equilibrium pressure and $\mathrm{R}$ is the ideal gas constant.

\subsubsection{Supersaturation}

At equilibrium conditions, described by equation 3.1, the crystal-vapor system is stable and no transformation will occur. However, when the external conditions are altered so that they can deviate the pressure and temperature from their equilibrium values, the system gets unstable and energy transfer begins in order to go back to a more stable state. Therefore, epitaxial growth is a non-equilibrium process. As can be seen in the phase diagram (Fig.3.1), taking as a reference the equilibrium temperature $T_{0}$, when $P>P_{0}$ the crystal phase becomes stable and the growth from vapor to solid can take place. This also means that the chemical potential of the crystal phase is smaller than the chemical potential of the vapor phase, as can be seen in Figure $3.1 \mathrm{~b}$. On the contrary, if $P<P_{0}$ the chemical potential of the crystal is higher than the one of the vapor and instead of growth there would be sublimation. Therefore, the thermodynamic condition for the growth to occur is that $\mu_{c}<\mu_{v}$. The difference in chemical potentials between crystal and vapor phases is the driving force for the growth to happen, which is also known as supersaturation since the vapor phase has to be supersaturated to fulfill the chemical potential condition. At a given pressure, $\mathrm{P}$, the supersaturation can be expressed as:

$$
\Delta \mu^{s}=\mu_{c}(P)-\mu_{v}(P)
$$

Equation 3.7 can be written as:

$$
\Delta \mu^{s}=\left[\mu_{c}(P)-\mu_{c}\left(P_{0}\right)\right]-\left[\mu_{v}(P)-\mu_{v}\left(P_{0}\right)\right],
$$



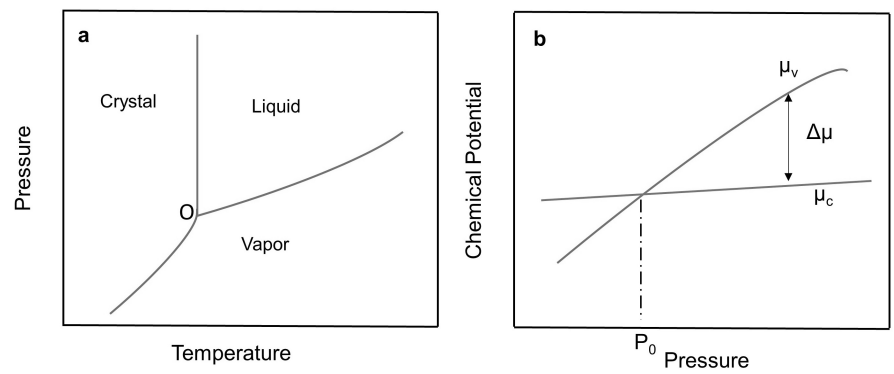

Figure 3.1: a) Phase diagram simplified for a single component system, b) Chemical potential variation with pressure.

where $P_{0}$ is the pressure at equilibrium. Integrating from $P_{0}$ to $\mathrm{P}$ and substituting the molar volume of the vapor phase by the expression for ideal gas, results in:

$$
\Delta \mu^{s}=k \ln \frac{P}{P_{0}}
$$

Equations 3.8 and 3.9 describe the driving force of epitaxial growth from a vapor ambient phase in the general case. The work described in the thesis concerns the epitaxial growth of $\mathrm{GaN}$ from a vapor phase (Section 3.2). Therefore, at certain growth conditions of pressure and temperature the supersaturation, $\Delta \mu^{s}$, can be expressed as a function of the equilibrium vapor pressure of $\mathrm{Ga}$ and the experimental input partial pressure of $\mathrm{Ga}$ $[64,65]$ :

$$
\Delta \mu^{s}=\frac{P_{i n}^{G a}-P_{0}^{G a}}{P_{0}^{G a}}
$$

For practical applications it is important to know the exact value of the supersaturation for specific experimental growth conditions since it is directly related to the quality of the epilayer (for further details see Section 3.1.2). The calculation of supersaturation for $\mathrm{GaN}$ homoepitaxial growth is described in Chapter 6.

\subsubsection{Microscopic aspects of crystal growth}

From an atomistic point of view, atoms coming from the vapor phase have to reach the crystal surface and get adsorbed on it. The surface typically encountered by atoms consists of steps of different heights. Considering monoatomic steps for simplicity, one can see the step as a boundary region between the substrate surface and a vicinal surface separated by the equivalent length of an interplanar spacing. Those steps contain energetically different surface positions and atoms coming from the vapor phase will preferentially adsorb on 


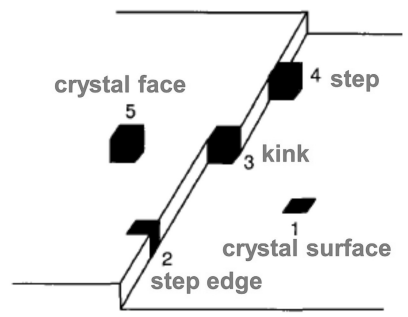

Figure 3.2: The step as a boundary between crystal surface and crystal plane [35].

the lowest energy possible position. During the processes of adsorption/desorption the number of dangling bonds of a specific surface position changes with the corresponding change of the surface energy. In Figure 3.2, position number 3 has a peculiarity since this is the only position in which the adsorption and desorption of an atom does not change the surface energy. Those particular positions are called kinks or half-crystal positions $[66,67]$ and play and important role in crystal growth. When the crystal is in equilibrium with its ambient phase the probabilities of adsorption and desorption from a kink position are the same. The process of crystal growth takes place at the interface defined by the crystal substrate and the vapor phase. The microscopic characteristics of this surface determine the crystal growth from the vapor phase. The crystal surfaces can be classified as a function of their number of unsaturated bonds, or dangling bonds, and therefore as a function of their specific surface energy, which depends directly on the density of unsaturated bonds. Therefore, the crystal surfaces can be flat (F), which corresponds to closely packed saturated surface as the c-plane of Ga polar GaN (ооо1), stepped (S) which are non-closed packed with higher density of kinks and finally kinked (K) surfaces, which relates to a surface with a significant amount of dangling bonds and maximum density of kink positions. The surface free energy increases with the number of unsaturated bonds, therefore the F surfaces will be more stable than the K surfaces since they have lower surface energy. In addition, the growth velocity of $\mathrm{K}$ faces will be higher than for the $\mathrm{F}$ faces, due to the availability of kink positions. For instance, $\mathrm{K}$ faces are the ones which disappear first followed by $S$ faces. The close packed surfaces, the $F$ faces, are the ones which will remain after a while. This is particularly important when it comes to reformation of nanowires and for the equilibrium crystal shape under different growth conditions.

Crystal surfaces can be also classified as a function of the angle with respect to the direction where the most dense row of atoms is localized. A singular face has 0 degree 
angle while a vicinal face has a certain off-cut angle different from 0 . This fact has to be taken into account since the cutting of substrates with a certain size always implies a deviation from the direction of the close packed face. A vicinal surface always provides step-like surface morphology. The step height and the terrace length depend directly on the off-cut angle [68]. At the crystal-vapor interface, the energy invested to form a kink is $\psi / 2$, where $\psi$ is the bond strength determined by the types of atoms in the crystal lattice. The density of kinks at a particular step, $\eta$, is defined as the number of kinks, $n$, over the smooth parts on the steps, $n_{0}$. According to the theory of Burton, Cabrera and Frank [66], the density of kinks are related to the bond strength and temperature by:

$$
\eta=\exp \left[\frac{-\psi}{2 k T}\right]
$$

From the above equation one reads that the formation of kinks is activated by thermal energy. The mean distance between kinks, $\delta_{0}$, also depends on the temperature and the bond strength through:

$$
\delta_{0}=a\left[1+\frac{1}{2} \exp \left(\frac{\psi}{2 k T}\right)\right],
$$

where a is the first neighbour atomic distance in the step. Hence, at elevated temperature $\delta_{0}$ decreases which means that there are more kinks. From a thermodynamic point of view the crystal face roughening, i.e., the equilibrium crystal shape, is defined through the Gibbs free energy of a step:

$$
G_{\text {step }}=-n_{u} k \ln \left(\eta \frac{1+\eta}{1-\eta}\right)
$$

Where $n_{u}$ is the number of the unsaturated bonds normal to the step. The temperature always induces disorder, so that the entropy of the system increases:

$$
G_{\text {step }}=U_{\text {step }}-T S_{\text {step }}
$$

The Gibbs free energy of the step increases in absolute value with the number of kinks on the face. The opposite force in this case is the potential energy of the unsaturated bonds at the step, $U_{\text {step }}$ which is given by:

$$
U_{\text {step }}=\left(n_{u}+2 n\right) \frac{\psi}{2}
$$

When the $\mathrm{T}$ is sufficiently high, the entropy term of equation 3.14 wins over the potential energy, and the Gibbs free energy gets a negative value. This $\mathrm{T}$ is called critical temperature 
and its value is inferred from the equilibrium condition $\Delta \mathrm{G}_{\text {step }}=0$. Above the critical temperature the atomic steps disappear giving rise to roughening of the faces. Below such temperature, the steps can get rough but still exist.

Therefore, the roughness of a crystal face, $\mathrm{R}_{c}$, depends on its internal potential energy, $U$ at $T>0 K$, and $U_{0}$ at $T=0 \mathrm{~K}$.

$$
R_{c}=\frac{U-U_{0}}{U_{0}}
$$

At temperature of $0 \mathrm{~K}$ the crystal face is atomically smooth with only certain dangling bonds corresponding to its reconstruction. At $T>0$ some atoms on the surface have enough energy to abandon their position and leave vacancies. Those atoms can be adsorbed on another place creating an unsaturated lateral bond with the corresponding degree of roughness. Understanding crystal surfaces is important since they affect the growth mechanism. The step free energy, which mainly depends on vertical and lateral unsaturated bonds, should not be confused with the specific surface energy which depends on the specific rearrangement of atoms within a certain crystal facet. In that regard, more closedpacked faces have lower specific surface energy since they have more density of bonds within the crystallographic plane so that in equilibrium its stable surface morphology will be flat while in the case of less close-packed faces will be rounded [69, 70].

\subsubsection{Nucleation}

In the unstable supersaturated phase, in our case the vapor, the density of active species like molecules and radicals is not perfectly homogeneous across the entire phase volume. There will be certain number of fluctuations which lead to a "heterophase" susceptible to produce aggregates when $\mu_{v}>\mu_{c}$. In other words, beyond a critical supersaturation, the unstable vapor phase required for the growth will start producing small clusters of building units, atoms, known as a nuclei. The nucleation process is governed by the interaction between growing layer and substrate. When active species travel through the vapor phase towards the proximity of the substrate surface, they are subjected to the surface potential energy. Every surface has a specific surface energy, which is directly related to its reconstruction and therefore to the number of dangling bonds. In addition, the strain has an influence on the surface energy since it rises the chemical potential. Due to the single-crystal nature of the substrate, and therefore its periodicity, the potential energy will be a periodic function with a period corresponding to the spacing between the atoms and with an amplitude equivalent to the surface energy desorption [35]. The interaction between the epilayer and the substrate is given by the wetting function, $\phi(\theta)$ :

$$
\phi(\theta)=\frac{1}{4}\left[(1-\cos \theta)^{2}(2+\cos \theta)\right],
$$


where $\theta$ is the contact angle between the nuclei and the surface of the substrate. When the wetting is complete the interaction is maximum, and a $2 \mathrm{D}$ nucleus will form. For a total non-wetting the substrate does not interact with the epi-layer, thus the nuclei would be $3 \mathrm{D}$ and also strained. The latter is also called homogeneous nucleation. Heterogeneous nucleation corresponds to the situation when the interaction between substrate and nuclei is not so large to lead to a complete wetting and it is not so small to have a total nonwetting. In other words, the wetting of the substrate by the deposited GaN depends on the interatomic forces between substrate and nucleus. The strain state of the nuclei plays an important role for the nucleation scheme. For a pure homogeneous nucleation, where $\phi(\theta)=1$, the $3 \mathrm{D}$ nuclei is strained. The chemical potential of the nuclei, and therefore of the new crystal phase being formed increases according to:

$$
\mu_{c}^{\text {strained }}=\mu_{c}+2 \epsilon,
$$

where $\epsilon$ is the strain of the nuclei. Since $\Delta \mu=\mu_{v}-\mu_{c}$, the increase of the nuclei chemical potential leads to a reduction in the overall supersaturation. For lower strain state the nuclei turns to be $2 \mathrm{D}$. In the ideal case with no lattice mismatch, i.e., in homoepitaxy, the $2 \mathrm{D}$ nucleus would be unstrained. The chemical potential of the $2 \mathrm{D}$ nuclei is lower than the chemical potential of the ${ }_{3} \mathrm{D}$ nuclei, which leads to a larger $\Delta \mu$ at a given $\mu_{v}$. It follows that the equilibrium vapor pressure of the $2 \mathrm{D}$ nuclei is equivalent to $\Delta \mu$ while in the case of strained ${ }_{3} \mathrm{D}$ nuclei it is directly proportional to the strain.

$$
P_{\text {strained }}=P \cdot \exp \left(\frac{2 \epsilon}{k T}\right)
$$

Since the formation energy increases with the strain, the work employed in the formation of a $3 \mathrm{D}$ nuclei will be higher than for the $2 \mathrm{D}$ nuclei.

$$
\Delta G_{3 D}>\Delta G_{2 D}
$$

The critical size of a $3 \mathrm{D}$ nuclei, $n_{3 D}^{*}$, above which the nuclei can survive and start clustering, is proportional to the Gibbs free energy of formation and it is inversely proportional to the supersaturation [35]

$$
n_{3 D}^{*}=\frac{2 \Delta G}{\Delta \mu}
$$

Therefore there is a direct correlation between critical size of nuclei and supersaturation. In a similar way, the critical size of a $2 \mathrm{D}$ nuclei can be calculated from the exchanged energy during its formation:

$$
\Delta G_{2 D}=\frac{2 \chi^{2} s_{c}}{\Delta \mu-s_{c} \Delta \sigma},
$$




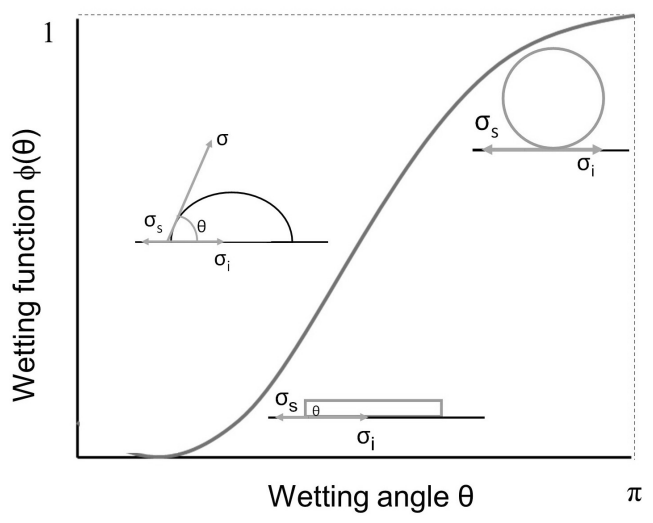

Figure 3.3: Nucleus shape for different values of the wetting angle $\theta$.

where $\chi$ is the specific edge energy and $s_{c}$ is the area occupied by a surface atom. $\Delta \sigma$ measures what is the dominant interaction and corresponds, according to Figure 3.3 to:

$$
\Delta \sigma=\sigma+\sigma_{i}-\sigma_{s}
$$

where $\sigma_{s}$ is the term which expresses the strength of the interaction between substrate's surface and nuclei. Therefore when $\Delta \sigma<0$ the force exerted by the substrate is strong and in theory, $2 \mathrm{D}$ nuclei could be formed although the environment is undersaturated. Thus, 2D nuclei can be formed also on foreign substrates. When $\sigma_{i}=0$ we have the case of homoepitaxy, the energy associated with the interface is 0 since both the epilayer and substrate are the same material. In this case $\sigma=\sigma_{s}$ and hence $\Delta \sigma=0$ and only 2D nuclei are thermodynamically favoured. Further details on homoepitaxy and heteroepitaxy are explained in section 3.2. Since the critical size of the nuclei increases while increasing its formation energy, we have:

$$
n_{2 D}^{*}=\frac{\Delta G}{\Delta \mu}
$$

Substituting equation 3.22 into 3.24 results in:

$$
n_{2 D}^{*}=\frac{2 \chi^{2} s_{c}}{\Delta \mu\left(\Delta \mu-\Delta \sigma s_{c}\right)}
$$



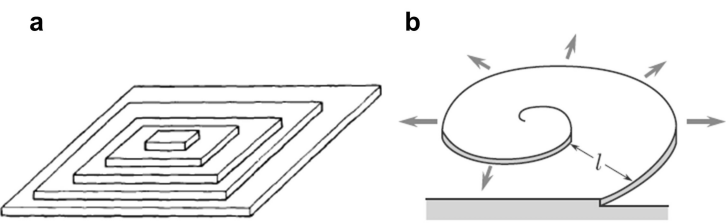

Figure 3.4: a) 2D nuclei clustering which forms a pyramid as growth advances [35] and b) step due to a spiral growth around a screw dislocation [71].

\subsubsection{Kinetic considerations}

Crystal growth takes place due to advance of steps. Steps are formed as a consequence of clustering of nucleus which have reached and overcame their critical size under given conditions. The origin of the steps depends on nucleation scheme which is directly related to surface specific energy. The growth theory [66] establishes that when there are enough screw dislocations terminating on the surface, these will be active sites for adatom incorporation and the initial growth stages will be in the form of spirals (Figure $3.4 \mathrm{~b}$ ). The origin of the steps in this case is the screw dislocations. On the contrary, when the defects of the substrate are not so abundant, the steps are formed as a consequence of clustering and advancement of initial 2D nuclei which adopt a pyramid shape (Figure 3.4 a). In growth from vapor phase there is an energy barrier $\Delta U$ at the boundary phase. This barrier can be associated with the activation energy of the chemical reactions, the products of which are the active species to be incorporated. Typically, these chemical reactions are activated by temperature. The probability to find a kink position is given by the ratio between the the adsorption sites along the step edge, $a$, and the mean distance between kinks, $\delta_{0}$. The surface diffusion energy of the adatoms, $E_{s d}$, depends on the periodic potential function of the substrate and in the ideal case of defect-free substrate, the surface diffusion is maximum. There is a competition between two processes when it comes to kinetic considerations. On one hand, the kinetic coefficient of the step, $\beta_{s t}$, is given by:

$$
\beta_{s t}=a v \frac{a}{\delta_{0}} \exp \left(\frac{-\Delta U}{k T}\right)
$$

On the other hand, we have adatom diffusion on the step which is regulated by the diffusion coefficient, $D_{s}$, and the mean distance, $\lambda_{s}$, via:

$$
\frac{D_{s}}{\lambda_{s}}=a v \cdot \exp \left(\frac{-\left(E_{d e s}+E_{s d}\right)}{2 k T}\right),
$$


where $v$ is the frequency of vibration of adatoms and $E_{\text {des }}$ is the adatom desorption energy. From equation 3.27 one reads that for a constant temperature:

$$
\left(\frac{D_{s}}{\lambda_{s}}\right)_{\text {GaNbulk }}>\left(\frac{D_{s}}{\lambda_{s}}\right)_{\text {GaN/AlN/SiC }}
$$

When $\frac{D_{s}}{\lambda_{s}}>>\beta_{s}$, the crystal face grows in a kinetic regime so the growth is limited by the kinetic advance of the step. On the contrary, when $\frac{D_{s}}{\lambda_{s}}<<\beta_{s}$, the crystal faces grow in a diffusion regime since the slow process in this case is the diffusion of the adatoms.

\subsubsection{Equilibrium shape of crystals}

Besides the growth of planar GaN epilayers oriented along the c-plane, we have also studied the reformation of GaN nanowires (NWs) (see for further details Section 3.3.). Here a brief introduction of the reformation process is presented in terms of the concept of equilibrium shape of crystals. Certainly, the shape of the growing structure has an influence on the equilibrium vapor pressure of the solid phase. For a hypothetical nuclei with surface energy $\sigma$ and vapor pressure $P_{r}$ growing on a planar GaN surface with vapor pressure $P_{\infty}$, the vapor pressure of the nuclei is greater than the vapor pressure of the infinitely large crystal. This reflects the differences in surface energies in the two cases. Once the nuclei has reached its critical size, it becomes unstable active structure where further incorporation of Ga adatoms producing clusters is favored. The influence of the surface energy on the equilibrium vapor pressure is described by the Thomson-Gibbs effect [35]:

$$
P_{r}=P_{\infty} \cdot \exp \left(\frac{2 \sigma V_{c}}{r k T}\right),
$$

where $\sigma$ is the surface energy, $V_{c}$ is the molar volume of the nuclei and $\mathrm{r}$ its radius. If $r$ is large enough then for $P_{r}=P_{\infty}$ there is no Thomson-Gibbs effect. Therefore, this effect is manifested only in nanostructures, e.g., in NWs. As a result of the Thomson-Gibbs effect the supersaturation is reduced and hence the growth rate would be also reduced. It is worth mentioning that the Thomson Gibbs effect takes place when the growth is not limited by mass transport. If the growth is limited by mass transport then the supersaturation and hence growth rate will be increased [72]. In our particular case the NW reformation takes place at lower temperatures compared to the growth of epilayers. Different crystal facets have different surface energies. In GaN, the c-plane (ooo1) is more closely packed than, for example, the m-plane (1-100) which results in lower surface energy of the c-plane compared to the m-plane. In other words, the c-plane is more stable. In that regard, the growth rate of each facet is also different, since it also depends on the surface energy. The reformation process consists of changing the initial NW shape as depicted in Figure 3.5 


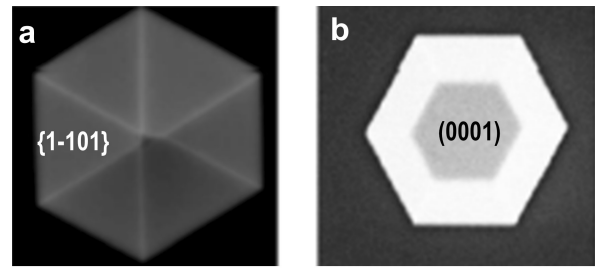

Figure 3.5: GaN facets: a) (1-101) and b) (ooo1) [70].

to a planar c-plane oriented GaN film. In order to ensure reformation, the experimental conditions must be tuned so that c-plane facet grows while $\mathrm{m}$-facets vanish. The driving force of the reformation process is the different chemical potentials of $\mathrm{Ga}$ of facets with different crystal orientations. Since the surface energy of the c-plane is lower that that of the m-plane, it follows that $\mu_{\mathrm{Ga},(0001)}<\mu_{\mathrm{Ga},(1-101)}$. The reformation takes place typically without any TMGa supply. We consider that the temperature and the volume are constant during the process and therefore, at equilibrium, the Helmholz free energy should be equal to 0 [35]:

$$
d F=0, d V=0
$$

Taking into account that the nanowires are immersed in the vapor phase, we have certain pressure in the vapor phase $P_{V}$ and in the crystal phase $P_{C}$. The energy and the area of a facet $n$ is represented by $\sigma_{n}$ and $\S_{n}$, respectively. The equilibrium condition is given by:

$$
-\left(P_{C}-P_{V}\right) d V_{C}+\sum_{n} \sigma_{n} d \S_{n}=0
$$

It is worth mentioning that at equilibrium conditions we have a volume part, related to the difference in equilibrium vapor pressure of both phases which does not depend on the crystal orientation, and a surface part which does depend on the crystal orientation. The volume of the crystal phase, $V_{C}$, is the same as the volume of the ensemble of nanowires, which can be written as:

$$
V_{C}=\frac{1}{3} \sum_{n} h_{n} d \S_{n}
$$

where, $h_{n}$ is the height of the $\mathrm{n}$-th nanowire. Since there are no Ga atoms coming from an external source, we have that any shift in the volume is equal to a variation of the height. Therefore an infinitesimal variation of the volume can be written as:

$$
d V_{C}=\frac{1}{2} \sum_{n} h_{n} d \S_{n}
$$


Substituting equation 3.33 into 3.31 one obtains:

$$
\sum_{n}\left[\sigma_{n}-\frac{1}{2}\left(P_{C}-P_{V}\right) h_{n}\right] d \S_{n}=0
$$

From Eq. 3.34 it follows that one of the terms should be equal to 0 . Since the area of a facet cannot be 0 , it follows that:

$$
P_{C}-P_{V}=2 \frac{\sigma_{n}}{h_{n}}
$$

The reformation process is driven by variation in surface orientation and $P_{C}-P_{V}$ is constant since the pressures do not depend on the crystallographic orientation. Therefore,

$$
\frac{\sigma_{n}}{h_{n}}=\text { const }
$$

This equation 3.36 provides the geometrical interpretation of the Wulff's theorem which gives a method to plot the equilibrium shape of a crystal under certain thermodynamic conditions [70, 73]. At this point the important aspect is how to predict which are the experimental conditions that stabilize certain crystal faces. The proportionality constant in Eq. 3.36 contains this information. The difference in equilibrium vapor pressures is equal to the variation of chemical potentials, i.e. to the variation of the supersaturation. Therefore:

$$
\Delta \mu^{s}=\frac{2 \sigma_{n} V_{C}}{h_{n}}
$$

Since $\sigma_{c-\text { plane }}<\sigma_{m-\text { plane }}$, according to Eq. 3.37 one would expect that the c-plane will be stabilized under lower supersaturation conditions, close to equilibrium. However, kinetics play also an important role. In the equilibrium crystal shape different facets can coexist and the facet with the slowest growth velocity will emerge.

\subsection{Homoepitaxy versus heteroepitaxy}

The difference between homoepitaxy and heteroepitaxy has its origin in the difference of chemical potentials between the substrate and the deposit. There are two main aspects to be considered:

- Nature and strength of the chemical bonds of the deposit and the substrate.

- Lattice parameters and crystal structure of the deposit and the substrate.

In the case of heteroepitaxy, the epilayer and substrate differ both chemically and structurally. On the contrary, in homoepitaxial growth, the epilayer and substrate are identical and therefore homoepitaxy is closer to crystal growth than to epitaxial growth. From a 
thermodynamic point of view, in epitaxial growth the difference in chemical potentials between the substrate and epilayer, $\Delta \mu \neq 0$ while in crystal growth $\Delta \mu=0$. In both heteroepitaxy and homoepitaxy, for the growth to take place, there should be a supersaturated vapor phase environment acting as a driving force. However, in the case of homoepitaxy since $\Delta \mu=0$ the system has to be pushed out of equilibrium and this has to be done by increasing the supersaturation. Therefore, higher supersaturated vapor phase is required for homoepitaxial growth. Another important difference is the catalytic influence of the substrate in the nucleation. The particles of the vapor phase feel a periodic potential of the substrate when they are close enough $[35,74]$. Therefore the nucleation scheme will be different. It is worth mentioning that, in the case of homoepitaxy the difference in chemical potentials between $\mathrm{GaN}$ substrate and $\mathrm{GaN}$ growing material, $\Delta \mu=0$, is not exactly the same. Impurity atoms, as well as dopant atoms change in a very subtle way the crystal lattice and therefore there will be a certain strain energy per atom. For instance, in this thesis Si doped GaN HVPE substrates are employed, and therefore when growing unintentionally doped $\mathrm{GaN}$ the chemical potential of this material would be slightly different than the chemical potential of the substrate. In addition, the concentrations of impurities $(\mathrm{C}, \mathrm{H}$ and $\mathrm{O})$ are different and can further contribute to the difference in chemical potentials. Strictly speaking, in homoepitaxy the small difference in chemical potentials, $\mu_{G a N, \text { deposited }} \neq \mu_{G a N, \text { substrate, }}$ is primarily due to the small lattice mismatch originating from the different strain energy per atom. Therefore, compared to heteroepitaxy, where lattice mismatches and chemical bonds strengths are very dissimilar, the difference in chemical potentials between the substrate and the deposit in homoepitaxy can be considered 0 .

$$
0 \approx \Delta \mu_{\text {homoepitaxy }}<<<<\Delta \mu_{\text {heteroepitaxy }}
$$

In heteroepitaxy, after the initial growth on the foreign substrate, subsequent growth takes place on the newly grown GaN material. Consequently, $\Delta \mu_{\text {heteroepitaxy }}$ will be reduced in comparison to the initial stage of growth. In our case, for growth on $\mathrm{SiC}$, a thin AlN nucleation layer of $\approx 50 \mathrm{~nm}$ is employed to reduce lattice and thermal expansion coefficient mismatches and to improve wetting. For the GaN thicknesses considered in this thesis (up to 2-3 $\mu \mathrm{m}$ ) the epilayer is still strained to some extent and hence $\Delta \mu_{\text {heteroepitaxy }}>\Delta \mu_{\text {homoepitaxy }}$ in the advanced stages of growth.

\subsection{Epitaxial approaches for reduction of defects}

To fully exploit the potential of GaN material for optimum device performance, defect and impurity incorporation during epitaxy needs to be controlled. The reduction of dislocation density and impurities is important since they have a direct impact on the 
breakdown voltage, leakage current and reliability of the devices. Theoretical studies have revealed that certain acceptor defects segregate along dislocation lines and are negatively charged [75]. Gallium vacancies $V_{G a}$ and other defect complexes, such as $V_{G a}-O_{N}$ and $V_{G a}-\left(O_{N}\right)_{2}$ act as deep acceptors [75]. Edge-type dislocations were shown to have a detrimental effect on electron mobilities and device performance [12, 13]. Therefore, in order to ensure an improvement of device performance, threading dislocation densities (TDD) must be minimized. The use of a foreign substrates for GaN integration limits the device design to lateral or quasi-vertical.

In this thesis two main strategies for defect reduction are studied. The first approach is based on reformation of GaN nanowires under conditions close to the thermodynamic equilibrium, which is presented in Paper I. This strategy offers an alternative to the use of expensive commercial GaN HVPE substrates. The defects coming from the substrate are filtered by the SiN mask, where small openings enable growth of nanowires. Any dislocations, which may propagate into the nanowires will bend and stop at the lateral facets of the nanowires, provided the openings are sufficiently small. Besides the cost reduction, the main advantage of this approach is the versatility in terms of substrates. The main limitation is that during the reformation process new defects may be generated, which require the optimization and controllability of the reformation process. A detailed study on the defects generated during reformation is presented in [76].

The emerging availability of GaN substrates, fabricated by the Ammonothermal and the Hydride Vapor Phase Epitaxy (HVPE) methods, has made it possible to develop homoepitaxial growth of GaN [21, 23]. In Paper II, we discuss the homoepitaxial growth by hot-wall MOCVD and the respective dislocation density reduction. The availability of $n^{+} \mathrm{GaN}$ HVPE substrates also enables the possibility to fabricate truly vertical GaN devices which is preferred regarding current distribution. Vertical device geometry allows for larger currents as compared to lateral geometry where the current is limited to a narrower space. In addition, in a vertical device the maximum electric field is situated far from the surface, which enables higher breakdown voltage. This is particularly useful when it comes to the development of medium-voltage devices above $1.2 \mathrm{kV}$ suitable for applications in e.g traction invertes in electric vehicles [6]. Typically, commercial GaN HVPE pseudo-bulk substrates have thicknesses of $300-400 \mu \mathrm{m}$ and threading dislocation densities in the range of $5 \times 10^{5}-2 \times 10^{6} \mathrm{~cm}^{-2}$ [25]. The TDD in GaN homepitaxial layers is reduced to $10^{5}-10^{6} \mathrm{~cm}^{-2}$ as compared to heteroepitaxial layers on SiC where TDD is $10^{8}-10^{9} \mathrm{~cm}^{-2}$. 


\section{Techniques for GaN growth}

\subsection{MOCVD}

The use of metalorganic precursors to grow group-III nitrides was reported for the first time in 1971 in an attempt to avoid the halide environment and make growth compatible to CVD systems [77]. Twenty years later the GaN technology was greatly advanced with the demonstration of $p$-type conductivity $[78,79]$ and the reduction of defect densities using AlN and GaN buffer layers. Metal organic chemical vapor deposition techniques (MOCVD) were one of the most used growth methods for large-scale GaN production in optoelectronics, power electronics, and for probe material in scanning microscope techniques [8o-82]. In any CVD process there are several sequential steps which are represented in Figure 4.1. First of all, the gaseous precursors are transported together with carrier gases from the bulk gas flow region to the reaction zone (step 1). The gas flow must be laminar (the gas front flows parallel to the substrate, as indicated in Figure 4.1) to allow

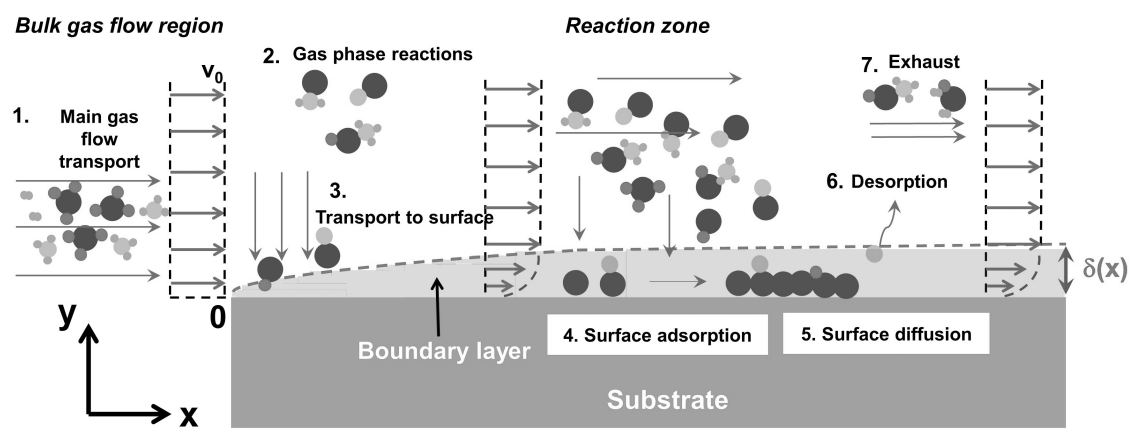

Figure 4.1: Velocity profile of the gas stream and main steps during CVD. 
the realization of next steps. Chaotic flows partially produced by elevated temperatures lead to flow patters in random directions, which would not let the active species to find the substrate. Once they reach the reaction zone, temperature activated chemical reactions such pyrolisis and dissociation, produce intermediate species like radicals which have the potential to activate subsequent reactions (step 2). The active precursors then travel towards the surface of the substrate (step3) where surface adsorption takes place (step 4). The adsorbed chemical species diffuse along the surface (step 5) until find an active adsorption site with dangling bonds available, like kink positions. Part of them are not adsorbed. Due to the thermal energy exchange some of the atoms get desorbed from the surface (step 6) with the subsequent delivery to the exhaust system (step 7) [83, 84]. The gas velocity is not constant across the reaction chamber. There is a gradient of gas velocity, directed normal to the substrate' surface, in such a way that the velocity at the origin point is 0 . The hard parts of the reactor, such as walls and satellite carrier, exert frictional forces to the gas which in fact slows it down. The boundary layer, which has a thickness dependant on the distance to the origin point, is the region where there is a gradient of gas velocity due its interaction with the surroundings. The thickness of the boundary layer, $\delta(x)$, is inversely proportional to the gas velocity, according to:

$$
\delta(x) \propto \sqrt{\frac{v x}{v_{0}}},
$$

where $v$ is the kinematic viscosity of the gas and $x$ the distance from the established origin. As in any other process, CVD is kinetically limited by the slowest step. The kinetics of the CVD process is determined by the growth temperature. There are three regimes in CVD processes [84]:

1. Low temperature regime: Within this regime the growth rate is limited by the kinetics of the reactions in the gas phase or at the substrate surface. By an increment of the temperature the active species have more energy to overcome the activation energy and therefore the growth rate increases.

2. Medium temperature regime: Here the active species have already enough energy to overcome the energy barrier and the slowest step is the diffusion across the boundary layer. The driving force for this step is the gradient of gas velocity within the boundary layer. Therefore, thinner boundary layer gives rise to a faster growth rate. From equation 4.1 we have that the higher the viscosity the thicker is the boundary layer whereas slower gas stream leads to thicker boundary layer. The gas viscosity is influenced by the carrier gas mixture composition. In this thesis for all the experiments a mixture of $\mathrm{H}_{2}$ and $\mathrm{N}_{2}$, with varying composition depending on 


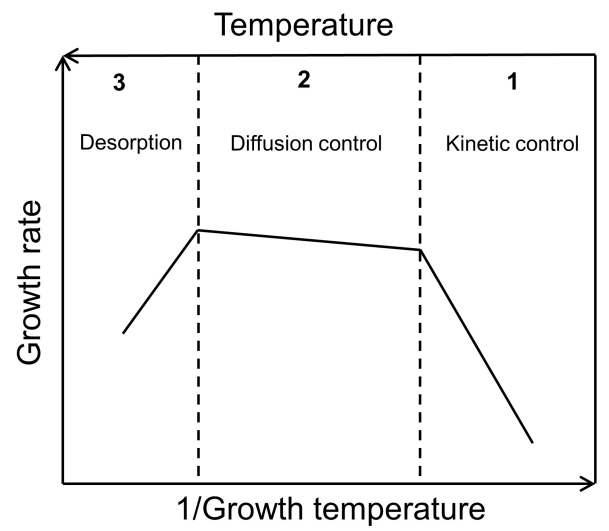

Figure 4.2: Growth regimes for different regions defined by growth temperature.

the desired growth result, has been used. For instance a nitrogen rich mixture is more viscous than a hydrogen rich mixture so the boundary layer gets thicker with the corresponding slower growth rate. The gas velocity depends on growth pressure. Low pressure favours laminar flow and the gas velocity increases, hence the boundary layer gets thinner. On the contrary, at high pressures gas phase reactions become important, laminar flow gets slower and the boundary layer becomes prominent. Therefore, within this regime, high growth pressure leads to slower growth rate. At very low reaction pressures only the surface reactions contributes to the growth. Therefore, in the intermediate regime one cannot work at a very low pressure since the diffusion through the boundary layer is not possible anymore.

3. High temperature regime: Completely the opposite to case 1 . Here the growth rate decreases with temperature due to desorption of reactants and activation of parasitic reactions. At high process temperature the gas phase reactions get activated and the partial pressure of the precursors also increases. Therefore a rise in temperature drops the supersaturation.

\subsubsection{Hot-wall MOCVD for GaN growth}

\subsubsection{Principles}

Hot-wall MOCVD provides homogeneous distribution of temperature which allows for better cracking efficiency of the precursors. It further allows for independent control of the 
gas phase chemistry over the substrate and the surface diffusion, which may be exploited to optimize the epitaxy for minimum incorporation of impurities in a wide growth window. In addition, due to this homogeneously heated environment, the formation of $\mathrm{N}$ radicals is possible, which can take part as intermediates in chemical reactions activating them. This "chemically activated environment" produces better yield of the reactions, but with the drawback of potential higher carbon incorporation. The concept of the hot-wall MOCVD was initially developed for the high temperature epitaxy of $\mathrm{SiC}$ [85]. Later on it was implemented for growth of AlGaN/GaN high electron mobility transistor (HEMT) structures on $\mathrm{SiC}[86]$ and a mobility above $2000 \mathrm{~cm}^{2} /(\mathrm{V} \cdot \mathrm{s})$ in hot-wall MOCVD AlGaN/GaN HEMTs without a conventional thick C- or Fe-doped buffer was achieved [87]. Initial studies on GaN homoepitaxial growth by hot-wall MOCVD have shown that a two-dimensional growth and smooth surface can be achieved by applying a two-step heating process that allows surface stabilization of the GaN substrate [88]. Recently, it has been reported the successful implementation of the hot-wall MOCVD approach in the growth of N-polar III-Nitride materials [89]. The metalorganic precursor which supply $\mathrm{Ga}$ atoms used in this thesis is trimethyl gallium, TMGa. N atoms are supplied from an ammonia, $\mathrm{NH}_{3}$, source. The metal-organic precursors used as sources of the desired material must be pure and volatile so that their vapor pressure is higher than the atmospheric pressure at room temperature [84]. The simplified chemical reaction for GaN formation is [90]:

$$
\mathrm{Ga}(a d s)+\mathrm{NH}_{3} \rightleftharpoons \mathrm{GaN}(\mathrm{s})+(a d s)+\frac{3}{2} \mathrm{H}_{2},
$$

where (ads) is the number of available adsorption sites. In order to form GaN, free adsorption sites are needed for the Ga adatoms to diffuse towards and bind with $\mathrm{N}$. Those adsorption sites are the outcome of etching by $\mathrm{H}_{2}$ which, besides taking part in the formation of $\mathrm{GaN}$, acts as a carrier gas. Equation 4.2 represents a quasi equilibrium process which can occur at a wide range of temperatures. The GaN etching by desorption of Ga adatoms leaving the free adsorption sites and Ga droplets is thermally activated and can be described by:

$$
G a N_{(a d s)} \rightarrow G a_{s}+(a d s)
$$

It is worth mentioning that ammonia decomposition also plays a significant role since it provides additional $\mathrm{H}_{2}$ and $\mathrm{N}_{2}$ being activated by temperature.

$$
\alpha \mathrm{NH}_{3} \rightleftharpoons(1-\alpha) \mathrm{NH}_{3}+\frac{\alpha}{2} \mathrm{~N}_{2}+\frac{3 \alpha}{2} \mathrm{H}_{2},
$$

where $\alpha$ is the dissociation coefficient of ammonia. 


\subsubsection{Reactor configuration}

In hot-wall MOCVD, the susceptor where a satellite carrier is placed in the middle allows the substrate for epitaxial growth to be surrounded by a heated environment. The susceptor is typically made of graphite, and it is coated by tantalum carbide $(\mathrm{TaC})$ to avoid chemical reactions of ammonia and by-products with graphite. In the outer part, there is an extra insulation system made of porous graphite equipped with gas lines where nitrogen is constantly supplied during growth to guarantee homogeneous temperature distribution. The heating is provided by a coil connected to a radio-frequency generator. Due to the shape of the reactor, the magnetic field envelops the entire substrate, preventing horizontal and vertical temperature gradients [85]. This is particularly useful to reduce bowing effects, since a temperature gradient in the vertical direction would produce different heat distribution at the bottom and at the top of the substrate, leading to bowing and strain. At the same time, the lack of strong temperature gradients in the horizontal direction ensures a more uniform concentration of gaseous species near the substrate [91]. The substrate is placed on top of a rotatory satellite carrier which rotates either by flowing $\mathrm{H}_{2}$ or $\mathrm{N}_{2}$. The rotation ensures homogeneous temperature distribution over the substrate. The hot-wall MOCVD reactor is a complex system with a specific purpose to deposit epitaxial layers on a substrate. It has many parts and components interacting with each other through different connections. In addition, there is a continuous flow of energy, materials and information since the system is programmed prior to the experiment. Inside the system there are continuous transformations between physical entities which flow through the reactor. For example, thermal energy is converted into chemical energy to break chemical bonds and to form new ones. Also there is a transformation of materials and phases; the precursors react in a gaseous state and the products are formed in a solid state.

As a complex system, the reactor also has several subsystems:

- Gas delivery system: Its purpose is to supply and control the flows of the precursors to the reaction chamber. The solid group-III source, e.g., TMGa, is contained in a bubbler. The bubbling gas, in our case $\mathrm{H}_{2}$, captures TMGa vapors bubbling through the liquid. The hydrogen flow coming into the precursor is controlled by a thermal mass flow controler (MFC). In the process of bubbling an equilibrium between vapor and liquid phases is established with the equilibrium vapor pressure being the main parameter. Since the vapor pressure strongly depends on temperature, the bubbler is immersed in a heated water bath at a constant temperature of $3{ }^{\circ} \mathrm{C}$. The $\mathrm{N}$ precursor, $\mathrm{NH}_{3}$, flows in the hydride line together with the carrier gases $\mathrm{H}_{2}$ and $\mathrm{N}_{2}$.

- Heating system: The susceptor is heated by a water cooled RF coil. The growth temperature is assessed by a pyrometer located in the top part of the susceptor 

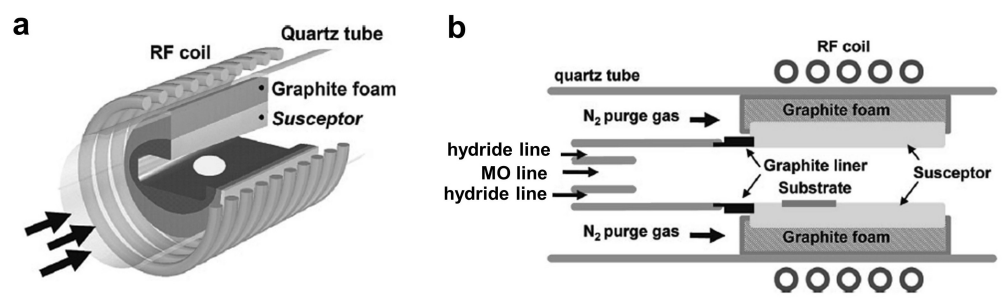

Figure 4.3: Configuration of the hot-wall MOCVD reactor used in this thesis [92]: a) Cylindrical shape of susceptor and b) Cross-section view of separated gas lines to avoid reactions before reaching the substrate area. Hydride lines contain: $\mathrm{NH}_{3}, \mathrm{H}_{2}$ and $\mathrm{N}_{2}$. MO line contains TMGa and carrier gases: $\mathrm{H}_{2}$ and $\mathrm{N}_{2}$.

and aligned before each growth run to guarantee constant position. The pyrometer provides feedback to regulate the RF power controlling the growth temperature.

- Pumping system: There are two vacuum pumps in the employed hot-wall MOCVD reactor. At first, the growth pressure is controlled by a mechanical pump with a throttle valve. Then the vacuum inside the reactor needed to start the growth run is accomplished by the use of a turbo pump. Typically, before each growth experiment the desired background pressure is in the order of $8 \times 10^{-6}$ mbar to ensure there is no residual gaseous water.

- Exhaust system: This system handles the by-products, residual unreacted gases and carrier gases passing through the vent line. It uses a scrubber device where all the residues are chemically treated prior to their waste.

\subsubsection{Impurity sources}

In spite of the fact that impurity incorporation can be reduced by tuning the growth conditions [93], there are several impurity sources which do not depend on them:

- Purity of metalorganic precursors and/or carrier gases.

- Surrounding surfaces of the reactor.

- Substrate surface.

During the high temperature MOCVD process point defects get enough mobility to redistribute in the bulk grown material and their generation and incorporation is governed by their formation energy. The latter depends on the partial vapor pressure of the 
gaseous species under certain experimental conditions. From the quartz parts of the reactor and from non-pure metal-organic precursors, Si can be present as an impurity. For our optimum homoepitaxial growth conditions, the Si concentration is $(2-5) \times 10^{15}$ $\mathrm{cm}^{-3}$, which is at the detection limit of secondary ion mass spectroscopy (SIMS). Oxygen incorporation is also common in MOCVD coming mainly from residual gaseous water and from non-pure gaseous precursors and diluent gases. In order to have comparable values of residual $\mathrm{O}$ between growth runs performed at the same conditions, the run is started when the background pressure of the reaction chamber is $(8-9) \times 10^{-6}$ mbar. The oxygen incorporation depends on the position of the Fermi level and the surface reconstruction [93]. The O levels in our GaN are (1-3) $\times 10^{15} \mathrm{~cm}^{-3}$ which are also at the SIMS detection limit. $\mathrm{C}$ is an abundant impurity in MOCVD as it mainly comes from the metalorganic precurssor cracking. $\mathrm{C}$ is problematic when it comes to power applications of $\mathrm{GaN}$ since it creates traps. $C$ is an amphoteric impurity since it can act as a donor, when $C$ substitutes $\mathrm{Ga}\left(C_{G a}\right)$, or as an acceptor, when $C$ substitutes a $\mathrm{N}$ atom $\left(C_{N}\right)$. In n-type material the latter is the most common one due to its lower formation energy [94, 95]. C incorporation and defect formation $\left(C_{N}\right)$ can be controlled via variation of chemical potential of $C$ in the final state defined by our experimental conditions with respect to an initial reference state. The energy consumed to form the substitutional defect is given by [65]:

$$
\Delta E_{C_{N}}^{f}=\Delta \mu_{N}-\Delta \mu_{C}
$$

The incorporation of $\mathrm{C}$ can be controlled with the growth conditions. For that, the understanding of the physics behind carbon incorporation is of a major importance and its interpretation is summarized below. It is well known that the main $C$ source in $\mathrm{GaN}$ is the organometallic gallium precursor: trimethyl gallium, TMGa. As a result of TMGa cracking, the methyl groups in combination with hydrogen form methane which spontaneously pyrolise activated by temperature [96] :

$$
\mathrm{CH}_{4} \rightleftharpoons \mathrm{C}+2 \mathrm{H}_{2}
$$

with equilibrium constant given by:

$$
K=\frac{P_{C} P_{H_{2}}^{2}}{P_{\mathrm{CH}_{4}}}
$$

When increasing the input flow of TMGa, the partial pressure of Ga increases with the corresponding increment of the partial pressure of methane. If the diluent gas mixture is rich in hydrogen, then the equilibrium of equation 4.6 is displaced to the left, contributing to the rise in the partial pressure of methane. To keep the value of $\mathrm{K}$ constant the 
equilibrium vapor pressure of $C$ has also to increase so that more $C$ gets into the lattice. To understand the effect of the carrier gas on the concentration of residual $\mathrm{C}$, one has to consider the decomposition of ammonia given by equation 4.4. From this equilibrium it is clear that $\mathrm{N}_{2}$ favors ammonia decomposition whereas $\mathrm{H}_{2}$ displaces the equilibrium to the left. If we now consider the equilibrium constant of the GaN formation described in equation 4.2 we have that:

$$
K=\frac{\left(P_{\mathrm{H}_{2}}\right)^{3 / 2} a \mathrm{GaN}}{P_{\mathrm{Ga}} P_{\mathrm{NH}}}
$$

So under conditions which facilitates $\mathrm{NH}_{3}$ dissociation the equilibrium partial pressure of $\mathrm{NH}_{3}$ increases so that the equilibrium vapor pressure of $\mathrm{Ga}$ has to decrease to keep the constant value of $\mathrm{K}$ according to equation 4.8. This implies a reduction of the vapor pressure of methane and the corresponding reduction in the vapor pressure of $\mathrm{C}$ to keep the constant value in equation 4.7. Thus, $\mathrm{C}$ incorporation is lower under $\mathrm{N}$-rich environment. On the contrary, the ammonia decomposition is much poorer under $\mathrm{H}_{2}$ rich diluent gas mixture [65], therefore the equilibrium vapor pressure of ammonia gets lower with the corresponding increment in Ga vapor pressure according to the equilibrium constant. This leads to lower supersaturation and higher $C$ incorporation. In summary, since $C_{N}$ is a point defect located in the $\mathrm{N}$ sublattice, $\mathrm{C}$ incorporation can be controlled through the chemical potential of nitrogen. For $\mathrm{N}_{2}$ as a carrier gas the growth front is N-rich so:

$$
\Delta \mu_{N_{2}}>\Delta \mu_{H_{2}}
$$

and

$$
\left[C_{N}\right]_{\exp }=\left[C_{N}\right]_{r e f} \exp \left(\frac{-\Delta \mu_{N_{2}}}{k T}\right)
$$

From equation 4.10 [65] one reads that for a large absolute value of the variation in chemical potential of nitrogen (i.e. lower equilibrium partial pressure of nitrogen) there should be less concentration of $C_{N}$ defects since their formation energy increases (see equation 4.5). For our $\mathrm{GaN}$ grown at the optimum supersaturation conditions, a carbon concentration of $5.3 \times 10^{15} \mathrm{~cm}^{-3}$ has been achieved.

\section{2 $\mathrm{MBE}$}

Molecular Beam Epitaxy (MBE) is a technique to grow epitaxial GaN films. In contrast to MOCVD, there are no chemical reactions involved. The precursors for GaN growth are liquid $\mathrm{Ga}$, immersed in an effusion cell which produces a flux of $\mathrm{Ga}$ atoms whereas nitrogen atoms usually come from a plasma source in the case of plasma-assisted MBE. In order for the beams to meet at the surface of the substrate without interferences in 
their path, MBE growth is done under high vacuum environment, usually the pressure during growth is in the order of $10^{-5}$ mbar. To ensure that the MBE chamber is free of contaminants, an ultra-high vaccum conditions with a background pressure of at least $10^{-8}$ mbar should be achieved before the growth starts. To monitor the growth in-situ during the experiment it is possible to include in the same MBE chamber a Reflection High Energy Electron Diffraction (RHEED) set-up, since the high vacuum conditions required for RHEED are compatible with the vacuum needed for MBE. RHEED is a surface sensitive technique, which is very useful for growers during MBE experiments. In RHEED, an electron beam is accelerated by a voltage of about $15.8 \mathrm{kV}$ and is directed onto the surface of the substrate or epi-layer at a glazing incidence angle of $2^{\circ}$ which, together with the large energy of interaction, allows surface sensitivity. A fluorescent screen is included in the MBE chamber where one can see the RHEED electron diffraction pattern. This allows growers to determine at which temperature the growth can start, corresponding to the temperature at which the surface reconstruction appears. For example, in case of having $\mathrm{Si}(111)$ as a substrate, one can anneal the substrate in the MBE chamber until the $7 \times 7$ surface reconstruction appears, indicating that epi-ready surface is achieved. In addition, after a growth of a few monolayers, the RHEED pattern provides information on the growth mode. Spotty RHEED pattern corresponds to rough surfaces possibly leading to island growth while streaky pattern corresponds to smooth surfaces. An important parameter to control in each MBE growth is the beam equivalent pressure (BEP), which is the pressure measured just below the substrate. For identical precursor fluxes, the BEP pressure should be kept constant. There are two main regimes which can be distinguished in GaN growth by MBE: Ga-rich and N-rich. Since in MBE the growth is limited by the flux of Ga atoms, instead of V/III ratio used in MOCVD, one discusses III/V ratios. In the Ga-rich regime an adlayer of Ga atoms is known to produce better surface morphology due to enhance surface diffusion. For this regime, the III/V ratio has values between 1 and 1.4. In the N-rich regime, the Ga adatoms have lower mobility resulting in rough surfaces. For each MBE system, it is useful to construct the so called growth diagram in order to clearly know where are the boundaries between both growth regimes. The main advantage of MBE compared to MOCVD is the high-purity GaN epilayers, and the possibility to grow more variety of alloys with for example transition metal nitrides like Scandium and Yttrium, since the sources for MBE can be solid metal sources while in MOCVD is not always possible to find an organometallic precursor for each desired metal. With RHEED patterns it is also possible to determine the polarity of the grown GaN material. Ga polar gives $2 \times 2$ surface reconstruction while $\mathrm{N}$-polar gives $3 \times 3$ reconstruction, as can be seen in Figure 4.4. A drawback of this technique is the slow growth rate which makes difficult to implement it for industrial purposes. 

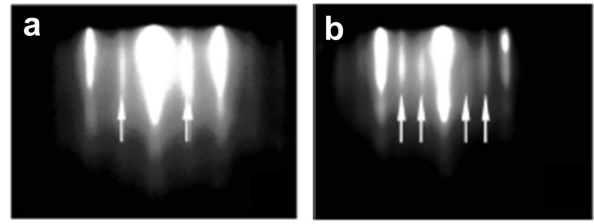

Figure 4.4: RHEED pattern of GaN grown by MBE with different polarities a) Ga-polar b) N-polar [97].

\section{$4 \cdot 3$ Sputtering}

Sputtering is an alternative method to grow $\mathrm{GaN}$ films in a high vacuum environment. Although it was initially employed to obtain policrystalline films of metallic alloys, nowadays epitaxial growth of group-III nitrides has been demonstrated. The growth by sputtering is a physical deposition method based on ejecting atoms from a target. The physical process of ejecting atoms has to be produced by accelerated particles. Those particles come from a plasma, usually Ar plasma, which is generated inside the growth chamber. The target is situated in a cathode and the substrate is placed in the anode. Depending on the applied voltage to generate the plasma, sputtering process can be: DC sputtering, where DC voltage is used, and RF sputtering employing radio frequencies. The sputtered atoms from the target travel through the vacuum chamber until reaching the substrate and get adsorbed on it. Radio frequency (RF) sputtering is used to deposit non-conductive materials so the charging of the target is avoided. The main parameters controlling the deposition and therefore directly affecting the material quality are: chamber pressure, substrate temperature, Argon pressure and bias voltage. The efficiency of sputtering was enhanced for deposition of materials at reduced temperatures by the use of permanent magnets situated around the target. The magnetic field induced by them localizes higher amount of active species from the plasma near the target, so the sputtering efficiency increases [98]. For GaN growth by sputtering, different substrates can be used such as $\mathrm{Si}$ and sapphire, where $\mathrm{N}_{2}$ gas was introduced together with Ar inside the deposition chamber. As a target, high purity metallic Ga can be used as well as liquid Ga. Due to the low melting point of $\mathrm{Ga}$, the experimental set-up might need adjustments such a horizontal cooled target where the sputtering gas can get into it. The growth by sputtering can be realized at lower temperatures compared with the growth by MBE or CVD. GaN grown on $\mathrm{Si}$ at a temperature of $450^{\circ} \mathrm{C}$ has been reported [99]. The sputtering technique enables high adatom mobility at low temperature due to the energy exchange between active plasma species and adatoms. Although it is expected that at reduced temperature 
the crystal quality of the material is not optimal, GaN epitaxial layers grown on sapphire have been demonstrated using as a target liquid $\mathrm{Ga}[100]$. 



\section{Characterization techniques}

\subsection{Optical microscopy}

In order to image transparent materials, such as group-III Nitrides, optical microscopes have to operate in a differential interference contrast (DIC) mode or Nomarski interference contrast (NIC). With NIC optical microscopy one can observe in a fast and simple way morphological features like large pits or cracks, which is important information needed to optimize growth conditions in epitaxy. The principle of the technique is based on the interference of two coherent beams, incoming and transmitted through the sample, of polarized light. This interference is sensitive to the optical path difference of the beams. The contrast is proportional to the path length gradient along the direction of the beams displacement. The difference in contrast give rise to a surface image which can be acquired under various magnifications.

\subsection{Atomic force microscopy}

Atomic force microscopy (AFM) belongs to the scanning probe microscopy techniques which are classified according to the probe-matter interaction. In AFM the interaction between the probe and the surface of the sample is through Van der Waals forces between instantaneous polarized atoms of the probe with the surrounding atoms [101, 102]. The magnitude of the Van der Waals forces depends on the distance of the interacting atoms, i.e the distance between probe and sample. Advantages of AFM with respect to other force microscopy techniques, such as STM, include the possibility to use electrically neutral samples and to do measurements at atmospheric pressure, which renders AFM fast, simple and cost effective. In AFM the probe is typically a micrometer-size tip which is attached to a cantilever. The cantilever is deflected as a function of the magnitude of the interaction between tip and surface. Surface imaging is realized by scanning the sample with the probe 


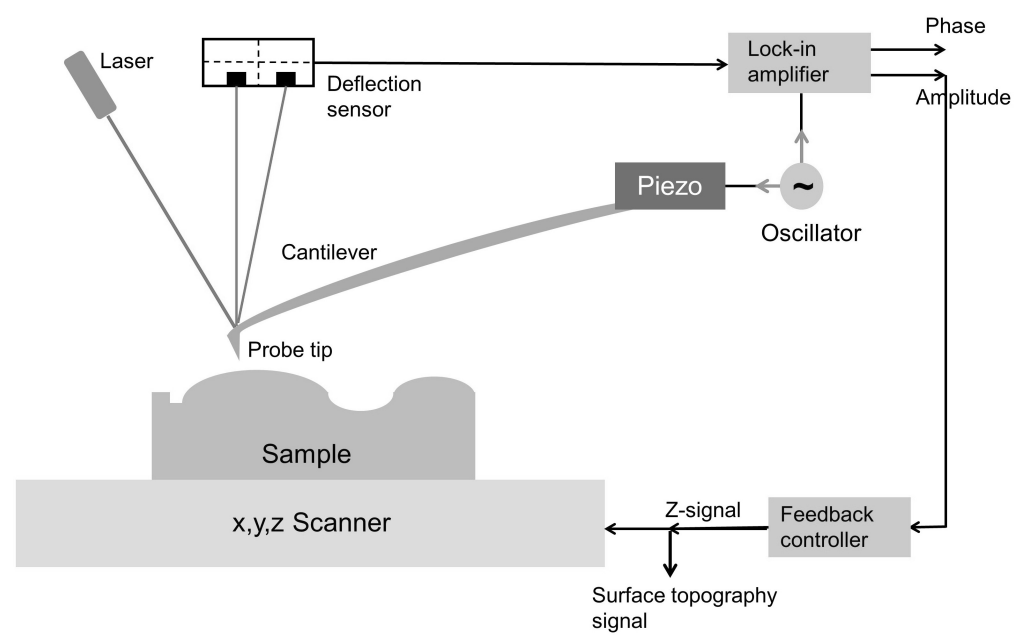

Figure 5.1: A schematic drawing of an AFM set-up.

tip digitalizing the deflection of the cantilever as a $z$-movement of the piezo as a function of the lateral position $x$ and $y$. An important characteristic of the Van der Waal forces is that they are very weak in the range of $10^{-11}$ to $10^{-6} \mathrm{~N}$ which allows a non-destructive imaging of the surface of the sample. Depending on the distance that separates tip and surface two modes of operation can be distinguished: contact and non-contact mode. In contact mode a small separation of the order of $\AA$, allows atomic resolution under best conditions. In contact mode, stronger forces due to short distance occur, such as ionic repulsion forces. On the opposite, in non-contact mode the tip-sample distance is in the order of hundreds of $\mathrm{nm}$. In this thesis, all the AFM measurements were done in tapping mode. This is a particular case of non-contact mode where the cantilever responses to the magnitude of the forces while probing the surface oscillating near its resonant frequency. The advantage of the tapping mode is that lateral probe-sample interactions are avoided. The oscillation of the cantilever is collected in form of amplitude by a laser light which is reflected on the backside of the cantilever that is detected by a position sensitive photodetector. 

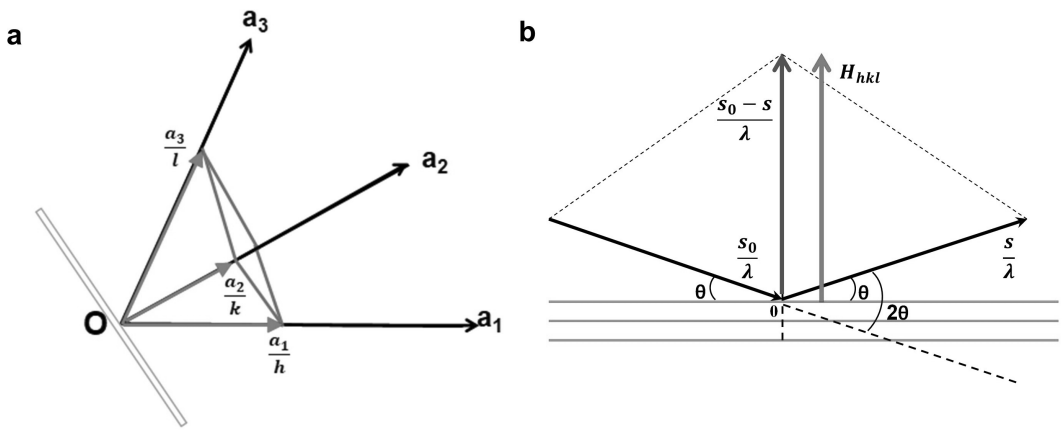

Figure 5.2: a) Miller indexes (hkl) describing a plane in real space, b) Construction of a scattering vector $\vec{H}_{h k l}$ in reciprocal space.

\subsection{X-ray diffraction}

\subsubsection{Basic principles}

$\mathrm{X}$-ray diffraction $(\mathrm{XRD})$ is a powerful non-destructive technique employed to determine the crystal structure of solid materials. XRD delivers information on the reciprocal space lattice, which can be used to deduce information on extended defect densities and residual strain in epitaxial materials, for example. In XRD an incident X-ray beam is scattered by the electrons of the atoms which form the crystal lattice. The electric field component of the X-rays electromagnetic wave (EMW) causes the electrons to oscillate and emit radiation with an energy equal to the initial X-ray EMW energy (elastic scattering process). A crystal is made of the repetition of unit cells which represent the smallest pattern of the crystalline structure. X-ray diffraction always gives information about the reciprocal lattice. If one constructs a base of vectors in real space, $\overrightarrow{a_{1}}, \overrightarrow{a_{2}}$ and $\overrightarrow{a_{3}}$ and takes a certain plane that intersects with $\overrightarrow{a_{1}}, \overrightarrow{a_{2}}$ and $\overrightarrow{a_{3}}$ at a certain points, $\frac{a_{1}}{h}, \frac{a_{2}}{k}, \frac{a_{3}}{l}$ those points are equivalent of the directions given by $\overrightarrow{a_{1}}, \overrightarrow{a_{2}}$ and $\overrightarrow{a_{3}}$ in reciprocal space. A plane in real space is a point in reciprocal space (Figure $5.2 \mathrm{a}$ ). The reciprocal lattice represents a mathematical formulation. A base of vectors in reciprocal space has the same meaning than in real space and can be constructed with vectors: $\overrightarrow{b_{1}}, \overrightarrow{b_{2}}$ and $\overrightarrow{b_{3}}$. The scattering vector is a position vector of the point with coordinates [hkl] in reciprocal space:

$$
\vec{H}_{h k l}=h \overrightarrow{b_{1}}+k \overrightarrow{b_{2}}+l \overrightarrow{b_{3}}
$$




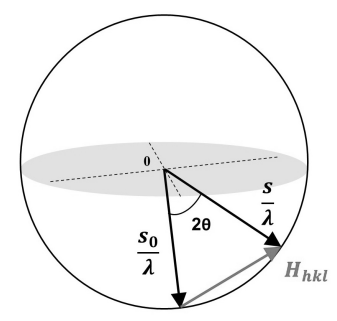

Figure 5.3: Diffraction condition represented by the Ewald sphere.

Since any distance in real space is equivalent to its inverse in reciprocal space, the magnitude of the vectors representing the incoming X-Ray beam, $\overrightarrow{s_{0}}$ and the elastically scattered $\mathrm{X}$-ray beam, $\vec{s}$, are multiplied by $1 / \lambda$. It is possible to see graphically in Figure $5.2 \mathrm{~b}$ that the path difference of both X-ray beams is equal to a vector normal to the plane containing the scattering center with its magnitude equal to the inverse of the interplanar spacing, $d_{h k l}$. Therefore:

$$
\frac{\vec{s}-\overrightarrow{s_{0}}}{\lambda}=\vec{H}_{h k l}
$$

where $\lambda$ is the wavelength of the $\mathrm{X}$-ray radiation used in the measurement. Equation 5.2 represents the Bragg's Law in a vector form [103]. The Physical meaning of the Bragg's Law is that the plane (hkl) will be detected only if a normal vector $\vec{H}_{h k l}$, which corresponds to the path difference of the initial and scattered $x$-ray beams, is a position vector of a point of the reciprocal lattice with reciprocal space coordinates [hkl]. The reciprocal lattice of the crystal is a mesh of points which are situated at the surface area of an imaginary sphere which is centered within a plane containing the sample under investigation. The fulfilment of the diffraction condition provided by the Bragg's Law can be represented by the so-called Ewald sphere of diffraction. With $1 / \lambda$ being the radius of the sphere and 0 the origin of the reciprocal lattice at a point where the incoming $x$-ray beam gets scattered, the scattering vector $\vec{H}_{h k l}$ would point to a position of the reciprocal lattice only if this point is situated at the surface of the sphere (Figure 5.3). In other words, the point [hkl] has to belong to the reciprocal lattice of the material under study to get diffraction signal from the corresponding (hkl) plane. 


\subsubsection{Kinematic theory of scattering}

The kinematic theory of scattering describes the changes of the EMW amplitude upon scattering by different centers. The scattering factor of an electron is given by:

$$
f_{e}=\int e^{\frac{2 \pi i}{\lambda}\left(s-s_{0}\right) r} \rho d V
$$

For an atom containing $\mathbf{n}$ electrons and assuming spherical charge density of the electron, the amplitude of elastic scattering per atom is given by the so-called atomic scattering factor:

$$
f=\sum_{n} f_{n}=\sum_{n} \int_{0}^{\infty} 4 \pi r^{2} \rho_{n}(r) \frac{\sin K r}{k r} d r
$$

Where $\mathrm{K}$ is:

$$
K=\frac{4 \pi \sin \theta}{\lambda}
$$

If we now consider as a scattering center a small crystal which is formed by many atoms, the resulting amplitude of the elastically scattered X-ray beam at a certain point of observation, e.g., the detector in the x-ray diffractometer setup is given by the structure factor, F:

$$
F=\sum_{n} f_{n} e^{(2 \pi i / \lambda)\left(s-s_{0}\right) r_{n}}
$$

The structure factor contains information about the atomic positions in the unit cell, therefore, it depends on the symmetry of the crystal structure. In other words, the elastically scattered radiation is directly related to the atomic position in the crystal unit cell and, thus, XRD can be used to retreive information on the symmetry group and the crystal structure of the material under investigation. The amplitude of a scattered wavefront is not a directly measurable quantity. Instead, one measures its intensity, which represents the square of the amplitude of the scattered radiation at a point of observation $\mathrm{P}$ and is given by:

$$
I=\frac{c E^{2}}{8 \pi}
$$

where

$$
\epsilon \epsilon^{*}=E^{2}
$$

with $\epsilon$ being the instantaneous electric field at that point and $\epsilon^{*}$ its complex conjugate.

\subsubsection{Calculation of threading dislocation densities}

If the crystal lattice were perfect, the reciprocal lattice would be formed by points. This is not the case in reality. The crystal lattice is distorted by defects such dislocations. XRD 
gives information about the perfection of the crystal lattice along the direction of the scattering vector $\vec{H}_{h k l}$. The result of those imperfections is the broadening of the reciprocal lattice points into ellipses [104]. The quantification of the broadening in reciprocal space can be used to deduce the type and density of dislocations in the material under study [105].

\subsubsection{Screw type dislocations}

The Burguers vector of screw dislocations, $b_{c}$, is parallel to the GaN (Ooo1) direction. Consequently, screw dislocations introduce broadening of the reciprocal points along [ool], or in other words causes mosaicity in terms of tilt of the crystallites. The broadening due to the tilt depends on the diffraction order because of the distortion field caused by screw dislocations. This allows to use the so-called Williamson-Hall plot to estimate the tilt angle from the $\beta_{\omega} \sin (\theta) / \lambda$ versus $\sin (\theta) / \lambda$ dependencies, where $\beta_{\omega}$ is the FWHM of the $\mathrm{GaN}(\mathrm{OOO} 2)$, (0004) and (ooo6) rocking curves [106]. From the linear fitting of the experimental data one extracts the tilt angle, $\alpha_{c}$. Taking into account the magnitude of the Burguers vector in the [0001] crystallographic direction, the screw dislocation densities is then given by:

$$
N_{S}=\frac{\alpha_{c}^{2}}{4.35 b_{c}^{2}}
$$

\subsubsection{Edge type dislocations}

Edge component dislocations comprise in-plane distortions along $\langle 11 \overline{2} 0\rangle$, which causes twist of the crystallites. Therefore, edge dislocation densities can be estimated from the twist angle and the magnitude of the Burguers vector, $b_{a}$, according to:

$$
N_{E}=\frac{\alpha_{a}^{2}}{4.35 b_{a}^{2}}
$$

For high dislocation densities above $10^{7} \mathrm{~cm}^{-2}$, the twist angle can be extracted following the model presented by Srikant et al., [107], using the tilt angle as a fitting parameter. The experimental data required is the rocking curve FHWMs of diffraction peaks measured at different inclination angles $\chi$. In the case of GaN-on-GaN material, the edge dislocations are reduced by two orders of magnitude compared to GaN-on-SiC material. Therefore, the above method does not provide reliable estimations and the twist angle is approximated by the FWHM of the (302) diffraction peak [105]. 
a

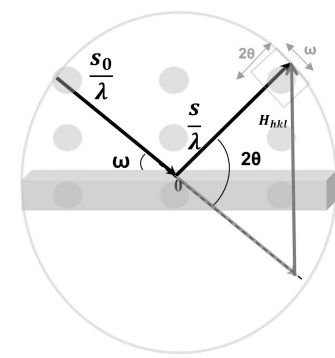

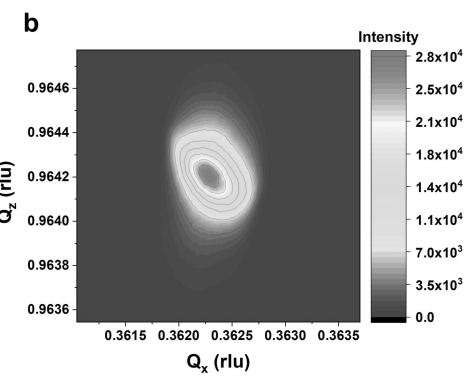

Figure 5.4: a) HRXRD and respective $\omega$ and $\theta$ directions in a RSM and b) RSM around the GaN 105 reciprocal lattice point for an HVPE GaN substrate.

\subsubsection{Reciprocal space mapping}

Reciprocal lattice points can be visualized by the simultaneous measurement of $\omega$ and $2 \theta$ scans. This measurement is a $2 \mathrm{D}$ map where both $\omega$ and $2 \theta$ angles are converted into reciprocal lattice units. Besides the qualitative observation of the reciprocal lattice point broadening, the RSM is a powerful tool to determine lattice parameters, and hence, in-plane and out-of plain strains. RSMs are typically performed using a high-resolution X-ray diffractometer set-up. Such diffractometer allows for various scans: $\omega, \omega-2 \theta, 2 \theta$, azimuthal, $\phi$-scans and tilt- or $\chi$-scans where $\omega$ is the angle between sample surface and incident beam ( $\omega=\theta$ in Bragg Bentano geometry), $2 \theta$ is the angle between incident and diffracted $x$-ray beams, $\chi$ is the tilt angle of the sample with respect to the sample normal and $\phi$ is the azimuthal rotation around the sample normal. RSMs can be used to map both symmetric and asymmetric diffraction peaks. In this thesis reciprocal space maps around the asymmetric $\mathrm{GaN} 105$ reciprocal point have been measured and analyzed to calculate lattice parameters and strain.

\subsection{Scanning electron microscopy}

Scanning electron microscopy (SEM) provides much higher magnification than the optical microscopy due to the shorter wavelength used compared to visible light [108]. Therefore, a more detailed information about surface topography can be obtained by SEM. An incident electron beam accelerated in the energy range from 0.1 to $30 \mathrm{keV}$ is used for imaging. When the incident electron beam hits the sample, there is an energy transfer to the electrons of the material under study which give rise to different secondary signals [109]. The initial electron beam penetrates the sample in a "pear shape" volume, the size of which depends 


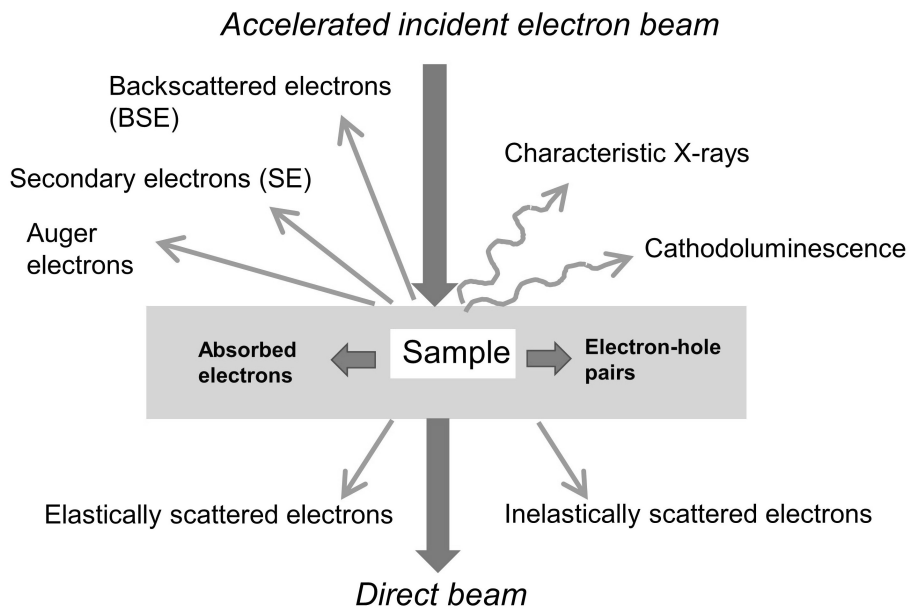

Figure 5.5: Different effects resulting from electron-matter interaction in an SEM.

on the atomic mass of the material constituents, on the angle of incidence and on the incident beam energy. In SEM the signals which are detected are: secondary electrons (SE) and backscattered electrons (BSE). Secondary electrons result from the excitation of an electron in the sample which uses part of the incident energy to get into an excited state. If a near-surface electron gets enough energy it can escape the sample surface and get into the detector. Typically, secondary electrons have energies of about $50 \mathrm{eV}$ and come from shallow areas about $1 \mathrm{~nm}$ below the sample surface [110]. SE provide very sensitive topographic information. Backscattered electrons have significantly higher energy than SE, and thus, are less surface sensitive. They arise from the interaction between the incident electrons with the inner core of the atoms of the sample and the scattering of electrons occurs at a high angle. Therefore the path of those electrons deviates in the opposite direction being able to escape from the sample and reach an energy selective backscattering detector. The imaging with BSE has less resolution than the one with SE, but provides instead information about the deeper regions of the sample and it is sensitive to i.e. compositional variations. For instance BSE energies depend on the atomic mass of the atoms and hence heavier atoms appear brighter than lighter atoms. The scattering process through a crystal lattice gives information about channeling effects, and therefore, structural information can also be obtained from the analysis of BSE. 


\subsection{Cathodoluminescence spectroscopy and imaging}

Cathodoluminescence (CL) is a contactless non-destructive technique that provides information about radiative transitions and luminescence distribution imaging. A CL set-up is commonly coupled to a SEM microscope so that the secondary electrons and the CL signal can be detected at the same time. In a SEM-CL measurement the same initial electron beam provides the energy to create the electron-hole pairs and the secondary electrons. Therefore two types of analysis can be done simultaneously: microscopy and spectroscopy. The microscopic analysis provides information about defect distribution and the spatial distribution of luminescence whereas spectroscopic analysis gives the energies involved in different radiative recombination processes. In CL spectroscopy a photon emitted as a result of a radiative recombination gives rise to the detected signal. The electron beam that hits the sample transfers energy which is partially invested in the excitation of electrons. This non-equilibrium state with an excess of carriers spontaneously goes back to the equilibrium state via electron-hole pair recombination. The photons emitted in the transition are detected as a CL signal. The recombination rate, $\mathrm{R}$, is directly proportional to a constant $\mathrm{B}$ and to the density of equilibrium concentrations of electrons and holes [63] according to:

$$
R=B n_{0} p_{0}
$$

The time electrons spend at the excited state before recombination is called recombination lifetime, $\tau$, which depends also on the excess of carriers. The higher the recombination time the shorter the corresponding lifetime.

$$
\tau=\frac{1}{B\left(n_{0}+p_{0}\right)}
$$

Depending on the nature of the energy levels involved in the transition there is intrinsic luminescence, when the energy levels are part of the natural state of the material like recombination across the bandgap, or extrinsic luminiscence when some energetic levels are introduced by defects or impurities. CL spectroscopy can be done either at room temperature or at low temperatures. Although the measurements at low temperatures require more complicated experimental set-up they allow the detection of excitonic transitions and infer relaxation mechanisms [111]. In Paper II room temperature CL spectroscopy is used to determine the band gap energy and obtain information about impurity incorporation. Different energy levels in the band gap of GaN are generated by various point and extended defects. For instance, dislocations can create either shallow levels coming from the strain fields or deep levels due to dangling bonds [63]. In addition, complexes resulting from vacancies associated with impurity atoms often contribute to the luminescence. The 
$\mathrm{CL}$ intensity in general increases with the increase of beam acceleration voltage. This means that when probing larger material volume more carriers are being generated and the dominant recombination process are of radiative type. However, quantitative analysis from CL intensities is not really feasible in many cases. For example, it is difficult to calculate accurately the concentration of carbon impurities, typically associated with the yellow band in GaN CL spectra, since the intensity is reduced by non-radiative recombination processes. CL mapping complements with additional information the area scanned with SEM. For example, by imaging non-radiative recombination centers the threading dislocation densities can be determined by dark-spot counting [112, 113].

In Paper II the dislocation densities of both GaN substrate before growth and GaN regrown layer have been estimated from the respective CL maps as a complementary technique to XRD. Some energy levels corresponding to strain fields induced by dislocations can be located very close to each other within the bandgap giving rise to non-radiative recombination processes which decrease drastically the CL intensity and hence appearing as dark areas. The strain fields around dislocations are weak and constitute a short range type interaction. Therefore, the dark spots appear in the vicinity of the dislocation centers which justifies their assignment to dislocations [114-116].

The SEM-CL analysis can be done in panchromatic mode, in which all light emitted by the sample is detected or in a monochromatic mode, when only the emission at a certain wavelength is detected. CL can also be applied in cross-sectional sample configuration to study the luminescence distribution along sample thickness [117]. The sample area from which CL signal comes is directly related to the acceleration voltage of the electron beam. At a higher acceleration voltage the beam has more energy to penetrate deeper into the sample increasing the density of electron-hole pairs and therefore increasing the recombination rate and the intensity. On the contrary, low currents of the incident beam results in a more surface sensitive measurement. Therefore the CL technique allows the realization of depth-resolved analysis. The sample volume probed at a different beam currents can be accessed by Monte Carlo simulations [118].

\subsection{Transmission electron microscopy}

Transmission electron microscopy (TEM) provides a local imaging with atomic resolution. The specimen under analysis should be thin in order to be transparent for the electron beam and to avoid inelastic and high angle scattering. Once the area of the material is chosen the lamella preparation for the TEM analysis is usually done by ion-milling technique. Details about specimen preparation can be found in reference [119]. A coherent beam of electrons is typically accelerated at energies from $30 \mathrm{keV}$ to $300 \mathrm{keV}$. The transmitted 
electron beams through the sample i.e., the direct beam, the elastic and the inelastic scattered beams (Figure 5.5) are then analyzed in order to construct respective images. The scattering processes have to be forward and coherent to give measurable TEM signal. Depending on the type of distribution of the scattered electrons it is possible to obtain different information. The contrast, generated by the scattered electrons allows to image the sample. Trough the angular distribution of the scattered electrons a diffraction pattern (DP) can be reconstructed. The DP is the distribution of diffracted electrons in reciprocal space so by TEM one can also obtain information about crystal structure, symmetry and quality. There are two main imaging modes in TEM. In the bright field mode (BF) only the direct beam is allowed to pass through the aperture located in the back focal plane of the objective lens [109]. The diffraction is affected by lattice imperfections such as dislocations. In addition, the contrast due to different atom mass and specimen density can be observed. In dark field (DF) mode, the direct beam is blocked by an aperture and only scattered beams are detected. Those beams contain a lot of information as a consequence of the strong interaction with the sample so one can study stacking faults, twinning defects and particle size among others. Different types of threading dislocations can be visualized in TEM by selecting the direction of the diffraction vector $\vec{g}$ which has to be parallel to the Burguers vector $\vec{b}$. So to visualize a screw dislocation in GaN the diffraction vector should be parallel to the [ooo1] direction. The invisibility criterion states that when the diffraction vector is normal to the Burguers vector the scalar product of both vectors is o $(\vec{g} \cdot \vec{b}=0)$ and therefore the dislocation will not be visible.

\subsection{Spectroscopic ellipsometry}

In Spectroscopic ellipsometry (SE) an electromagnetic wave interacts with a sample, which causes change of the polarization state of the incoming wave [120]. The electric field of the incident electromagnetic wave has two components: the s-polarized which is normal to the plane of incidence and the p-polarized mode is in the plane of incidence. Depending on the symmetry of the crystal structure and sample properties the optical response is different. For optically isotropic materials there is no conversion between s-polarized and p-polarized modes in the reflected (or transmitted) light. Optically isotropic samples can be analyzed by standard ellipsometry. In such cases the change of the polarization state of the incident electromagnetic wave after interaction with the sample is given by the ratio $\rho$ :

$$
\rho=\frac{B_{p}}{B_{s}}\left(\frac{A_{p}}{A_{s}}\right)^{-1}=\tan \Psi \exp (i \Delta),
$$




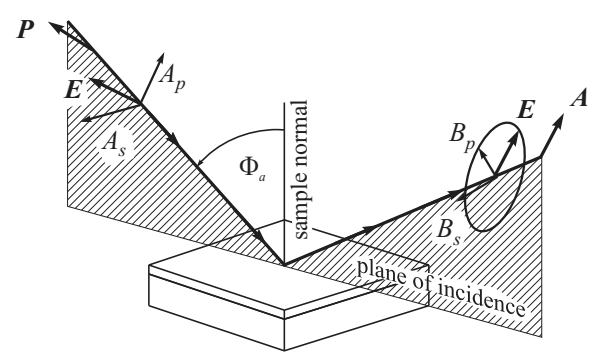

Figure 5.6: Schematic representation of spectroscopic ellipsometry measurement in reflection mode.
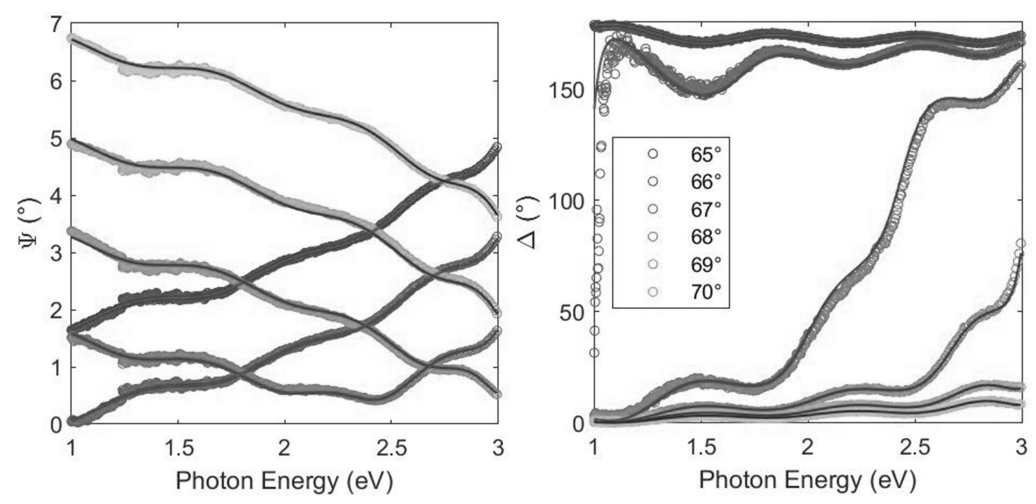

Figure 5.7: Measured (symbols) and modelled (lines) ellipsometric angles $\Psi$ and $\Delta$ for different angles of incidence (65-70 deg) around the Brewster angle. The small optical contrast between the UID layer and the $n$-type substrate causes layer interferences that allow accessing the layer thickness very precisely.

where $A_{p}, A_{s}, B_{p}$ and $B_{s}$ are the complex amplitudes of the $\mathrm{p}$ and $\mathrm{s}$ polarization modes before and after interaction with sample, respectively [121]. The SE parametrs $\Psi$ and $\Delta$ are defined through the ratio of the complex Fresnel reflection coefficients. The $\tan \Psi$ can be defined as the absolute value of the complex ratio, $\rho$, and $\Delta$ refers to the change in the relative phases of $\mathrm{p}$ and s polarized EMW components [122].

All samples studied in this thesis by SE are optically isotropic. In Paper II the thicknesses of unintentionally doped GaN layers grown on Si-doped GaN HVPE substrates were determined by UV-Visible SE. The measurements were done at several angles of incidence close to the Brewster angle to increase sensitivity to the refractive index difference [123]. The main reason for the difference between $\mathrm{GaN}$ substrate and $\mathrm{GaN}$ regrown layer 
refractive indices is due to the different doping. The ellipsometry data analysis is based on fitting the experimental data with a model. In our case the Cauchy model was used for fitting considering transparent materials with dispersive refractive index.

Figure 5.7 represents the experimental SE parametrs $\Psi$ and $\Delta$ for a homoepitaxial GaN on HVPE GaN substrate. When the sample presents in-plane optical anisotropy, there is a conversion between s-polarized light into p-polarized light and vice-versa. The optical response of the material under study is described then by generalized ellipsometry [120]. 



\section{Summary of main results}

\subsection{Nanowires reformation}

In Paper I we explore the potential of reformed GaN nanowires (NWs) fabricated by MOCVD for device-quality low-defect density templates and low-cost alternative to bulk GaN substrates. In contrast to epitaxial growth, NWs reformation occurs at conditions close to the thermodynamic equilibrium, which leads to a narrower window of experimental parameters. Figure 6.1 shows SEM images of different stages of the reformation process.

The effects of epilayer thickness and NW reformation conditions on the crystalline quality and thermal conductivity of the subsequent GaN epilayers are investigated. Thick GaN regrown layers on GaN reformed NWs templates, with thickness up to $10 \mu \mathrm{m}$ was achieved. The densities of screw and edge type dislocation were estimated from HR-XRD measurements. After GaN NWs reformation, the screw dislocation density is slightly reduced to $7.4 \times 10^{7} \mathrm{~cm}^{-2}$ with respect the value of the GaN/sapphire initial template of $8.5 \times 10^{7} \mathrm{~cm}^{-2}$. This confirms that NWs act as filters of dislocation with screw component. The lowest value is obtained for the thickest sample with a density of $3.8 \times 10^{7} \mathrm{~cm}^{-2}$. The surface morphology with respect to the reformed GaN material was also significantly improved with a rough square means $(\mathrm{rms})$ value of $0.24 \mathrm{~nm}$. Smooth surfaces with atomically step-like morphology with no spirals are achieved for $\mathrm{GaN}$ epilayers on the reformed NW templates, indicating step-flow growth mode. It is further found that annealing of the NWs at a temperature of $1030{ }^{\circ} \mathrm{C}$ in the presence of $\mathrm{NH}_{3}$ and $\mathrm{H}_{2}$, followed by a coalescence done at the same temperature under planar growth conditions, leads to the most efficient screw dislocation density reduction by nearly an order of magnitude. At these optimized conditions, the growth takes place in a layer-by-layer fashion, producing a smooth surface with a root mean square (RMS) roughness of $0.12 \mathrm{~nm}$. The highest thermal conductivity of $\mathrm{k}=206 \mathrm{~W} /(\mathrm{m} \cdot \mathrm{K})$, approaching the respective value of bulk $\mathrm{GaN}$, is obtained for the optimized 2- $\mu \mathrm{m}$-thick GaN layer. Our results suggests that NW reformed templates 


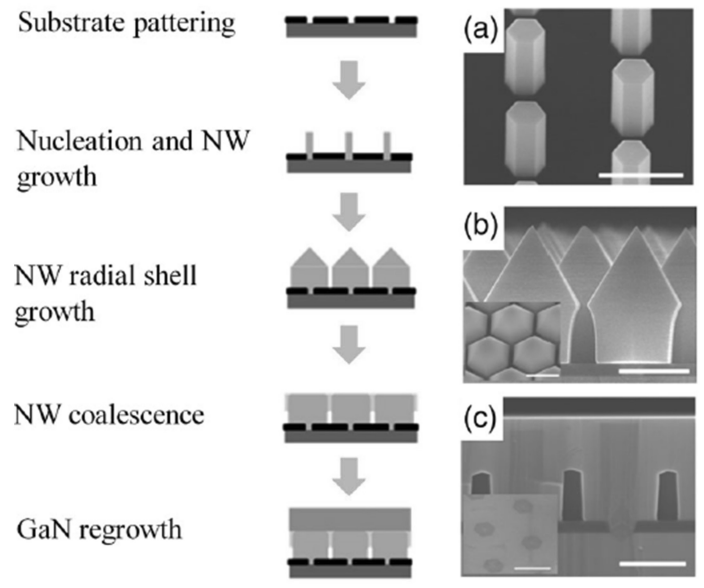

Figure 6.1: Schematic representation and SEM images of stages during growth and reformation of NWs. The scale bar in the SEM images is $200 \mathrm{~nm}$ [124].

could be a promising approach to reduce dislocation density in case of highly-mismatched substrates such as silicon.

\subsection{Homoepitaxial GaN growth}

Paper II presents a comprehensive study of homoepitaxial growth by hot-wall MOCVD. An indepth understanding of the homoepitaxial process from thermodynamic and kinetic points of view is detailed together with investigation of the thermal stability of GaN under different conditions. This allows us to select the optimum experimental conditions for growth and to avoid substrate degradation. It is proposed and experimentally demonstrated that a higher Ga supersaturation conditions are needed for homoepitaxy as compared to heteroepitaxial growth due to the difference in chemical potentials of the substrate and the epilayers.

We have found that for homoepitaxial conditions the surface quality is improved by using N-rich carrier gas mixture, i.e. at increased supersaturation, as can be seen in Figure 6.2 a. Growth under these growth conditions was shown to be within the mass-transport regime as deduced from the the growth rate dependence in Figure 6.2 b. 

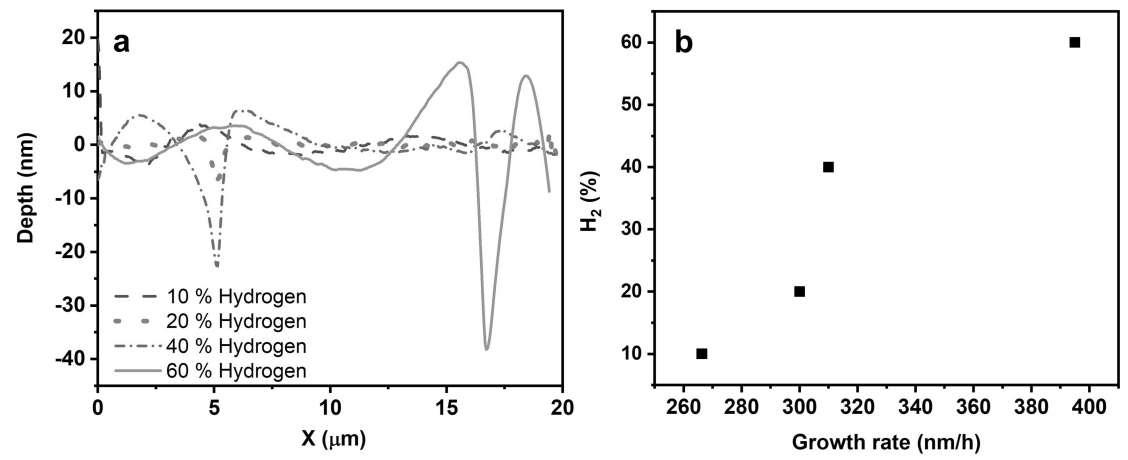

Figure 6.2: a) AFM profiles for GaN grown on GaN/AlN/SiC for several $\mathrm{H}_{2}$ composition b) Growth rate as a function of $\mathrm{H}_{2}$ composition.

We have shown that the initial growth instabilities (see AFM images in Figure 6.3), explained in terms of the ESB energy barrier [125], can be suppressed by increasing the growth rate, due to the alternative diffusion channel provided by the excess of Ga adatoms. The optimum homoepitaxial conditions, with a supersaturation value of $\sigma=3.4 \times 10^{4}$, allows us to achieve atomically flat GaN surface with an rms value of only $0.021 \mathrm{~nm}$ over a $20 \mu \mathrm{m}^{2}$ area (Figure $6.3 \mathrm{~b}$ ). The latter, to the best of our knowledge, is the lowest rms value reported for homoepitaxial GaN.

Investigations of GaN grown simultaneously on $\mathrm{GaN} / \mathrm{AlN} / \mathrm{SiC}$ templates and on GaN HVPE substrates reveal differences in growth mode. This indicates that the GaN materials of the template and the bulk substrate are not equivalent from kinetic and thermodynamic perspectives of crystal growth theory. The main difference in $\mathrm{GaN}$ grown on $\mathrm{SiC}$ (template) and GaN HVPE substrate is the residual strain. The residual strain of the $\mathrm{GaN}$ substrate can be considered negligible with respect to $\mathrm{GaN}$ on $\mathrm{SiC}$ since the thickness of the substrate is of the order of $350 \mu \mathrm{m}$. The $\mathrm{GaN}$ on SiC has a thickness of $700 \mathrm{~nm}$. Despite this value is above the critical thickness, there is still some residual strain in the layer. Consequently, the chemical potentials of the two GaN materials are different, which directly impacts the size of the critical nucleus and the specific surface energies. Another important difference between $\mathrm{GaN}$ template and $\mathrm{GaN}$ substrate is the screw dislocation density with $9 \times 10^{7} \mathrm{~cm}^{-2}$ for the GaN template and $1.1 \times 10^{6} \mathrm{~cm}^{-2}$ for the GaN substrate. The substrate surface has a catalytic influence on growth. The sites in the vicinity of screw dislocation terminations and impurity atoms have higher chemical potential. Therefore, the surface of the GaN/AlN/SiC template has higher specific surface energy i.e. higher catalytic effect than the surface of the GaN HVPE substrate. The wetting on the two 


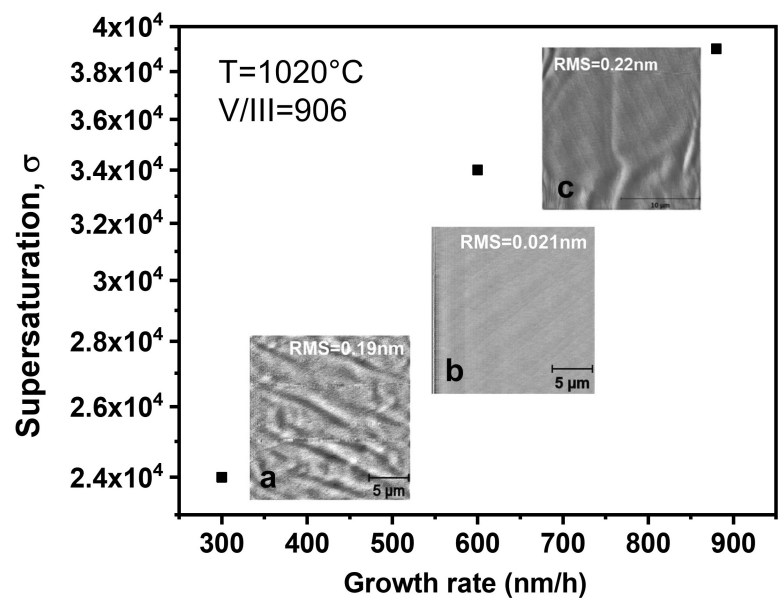

Figure 6.3: Supersaturation values vs growth rate for homoepitaxial $\mathrm{GaN}$ on $\mathrm{GaN}$ substrates with the corresponding AFM images.
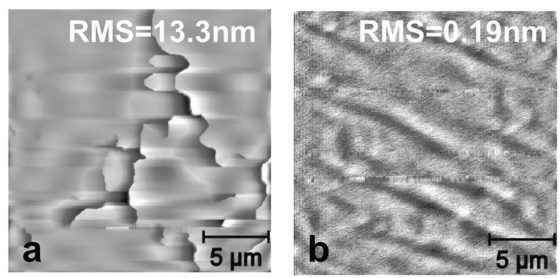

Figure 6.4: $20 \times 20 \mu \mathrm{m}^{2}$ AFM image of GaN grown at $1020^{\circ} \mathrm{C}$ on a) GaN/AlN/SiC template, $3 \mathrm{D}$ growth mode $b$ ) GaN HVPE, 2D growth mode.

different surfaces also is dissimilar: the wetting tends to be complete when GaN grows on GaN HVPE substrate while it gets poorer when the growth is on GaN/AlN/SiC. This has a direct implication on the nuclei formation: $2 \mathrm{D}$ nuclei are favoured on GaN HVPE substrate whereas strained ${ }_{3} \mathrm{D}$ GaN nuclei are formed on the surface of the GaN/AlN/SiC templates. Since the energy required for the formation of ${ }_{3} \mathrm{D}$ nuclei is higher than the one required for formation of $2 \mathrm{D}$ nuclei, more thermal energy is needed for GaN growth on $\mathrm{GaN} / \mathrm{AlN} / \mathrm{SiC}$ than for GaN-on-GaN growth. The growth kinetics is also different for the two types of GaN substrates since they have different morphologies i.e. step heights and widths. The energy required for surface diffusion is much lower when the growth occurs on GaN HVPE substrate than on GaN/AlN/SiC heterostructures. Therefore, at 


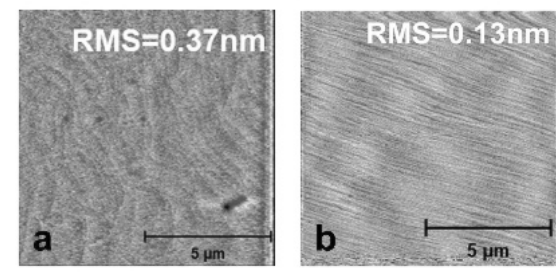

Figure 6.5: $10 x 10 \mu m^{2}$ AFM image of GaN grown at $1100{ }^{\circ} \mathrm{C}$ on a) $\mathrm{GaN} / \mathrm{AlN} / \mathrm{SiC}$ template b) GaN HVPE.

reduced growth temperature the growth on $\mathrm{GaN} / \mathrm{AlN} / \mathrm{SiC}$ occurs in $3 \mathrm{D}$ mode due to the poor diffusion while the $2 \mathrm{D}$ growth is preserved on GaN HVPE bulk substrate (6.4). The different GaN surfaces for template and bulk substrate leads to different growth regimes. For GaN-on-GaN the growth is limited by the kinetic advance of the step while the adatom diffusion is faster. On the contrary, when GaN grows on GaN/AlN/SiC the crystal faces grow in a diffusion regime and the growth is limited by the adatom diffusion, which is the slowest process in this case.

At high growth temperature of $1100{ }^{\circ} \mathrm{C}$ the growth mode is $2 \mathrm{D}$ on both $\mathrm{GaN}$ template and $\mathrm{GaN}$ substrate (Figure 6.5). At high temperature Ga adatoms diffuse faster leading to layer-by-layer growth mode on $\mathrm{GaN} / \mathrm{AlN} / \mathrm{SiC}$. At the same time, the kinetic advance of the steps which limits the GaN-on-GaN growth is also favoured at elevated temperatures.

Our results clearly demonstrates that although growth takes place on GaN material, this material on $\mathrm{SiC}$ is different from the material of the substrate in view of kinetics and thermodynamics. Even when growth of $\mathrm{GaN}$ on $\mathrm{GaN} / \mathrm{AlN} / \mathrm{SiC}$ advances to a thicknesses close to $1 \mu \mathrm{m}$, the situation is not equivalent of that for the homoepitaxial growth.

For the optimum value of supersaturation TDD densities are reduced with respect to the substrate achieving values of $4.5 \times 10^{5} \mathrm{~cm}^{-2}$ for screw type and $6.4 \times 10^{6} \mathrm{~cm}^{-2}$ for the edge type. Paper II contributes to the field in providing fundamental understanding about the different catalytic effect on the subsequent growth of the surface of the same material depending on properties such dislocation densities and strain. The paper also provides a quantitative information in terms of supersaturation which can be helpful for growers to design their own experiments. 



\section{References}

[1] E. Barrigón, M. Heurlin, Z. Bi, B. Monemar, and L. Samuelson, Chemical Reviews 119(2019).

[2] M. Khalilian, Z. Bi, J. Johansson, F. Lenrick, O. Hultin, J. Colvin, R. Timm, R. Wallenberg, J. Ohlsson, M.-E. Pistol, A. Gustafsson, and L. Samuelson, Small 16, 1907364(2020).

[3] F. Iacopi, M. V. Hove, M. Charles, and K. Endo, MRS Bulletin 40, 390 (2015).

[4] B. J. Baliga, Semicond. Sci. Technol. 28, 074011 (2013).

[5] T. Pu, Y. Usman, H. C. Chiu, K. Xu, H. C. Kuo, and X. Liu, Nanoscale Res. Lett. 16, 101 (2021).

[6] J. Hu, Y. Zhang, M. Sun, D. Piedra, N. Chowdhury, and T. Palacios, Mater. Sci. Semicond. Process. 78, 75 (2018).

[7] T. Kimoto, Jpn. J. Appl. Phys. 54, 040103 (2015).

[8] C. Gupta, Y. Enatsu, G. Gupta, S. Keller, and U. K. Mishra, Phys. Status Solidi A 213, 878 (2016).

[9] S. Kotzea, A. Debald, M. Heuken, H. Kalisch, and A. Vescan, J. Phys. D: Appl. Phys. 52, 285101 (2019).

[10] Y. Zhang, A. Dadgar, and T. Palacios, J. Phys. D: Appl. Phys. 51, 273001 (2018).

[11] J. T. Chen, J. Bergsten, J. Lu, E. Janzén, M. Thorsell, L. Hultman, N. Rorsman, and O. Kordina, Appl. Phys. Lett. 113, 041605 (2018).

[12] M. N. Gurusinghe and T. G. Andersson, Phys. Rev. B 67, 235208 (2003). 
[13] F. A. Marino, N. Faralli, T. Palacios, D. K. Ferry, S. M. Goodnick, and M. Saraniti, IEEE Trans.on Electron Devices 57, 353 (2010).

[14] C. A. Robertson, K. S. Qwah, Y.-R. Wu, and J. S. Speck, J. Appl. Phys. 126, 245705 (2019).

[15] H. Amano, I. Akasaki, K. Hiramatsu, N. Koide, and N. Sawaki, Thin Solid Films 163, 415(1988).

[16] H. Amano, T. Asahi, and I. Akasaki, Thin Solid Films 29, L205(1990).

[17] S. Nakamura, Japanese Journal of Applied Physics 30, L1705(1991).

[18] P. Gibart, Reports on Progress in Physics 67, 667(2004).

[19] T. S. Zheleva, S. A. Smith, D. B. Thomson, K. J. Linthicum, P. Rajagopal, and R. F. Davis, Reports on Progress in Physics 28, L5(1999).

[20] J. Colvin, R. Ciechonski, A. Gustafsson, L. Samuelson, B. J. Ohlsson, and R. Timm, Phys. Rev. Materials 4, 074603(2020).

[21] T. Paskova, D. A. Hanser, and K. R. Evans, Proc. IEEE 98, 1324 (2010).

[22] H. Fujikura, T. Konno, T. Suzuki, T. Kitamura, T. Fujimoto, and T. Yoshida, Jpn. J. Appl. Phys. 57, 065502 (2018).

[23] K. Grabianska, P. Jaroszynski, A. Sidor, M. Bockowski, and M. Iwinska, Electronics 9, 1342 (2020).

[24] T. Oka, Jpn. J. Appl. Phys. 58, SBo8o5 (2019).

[25] H. Fujikura, T. Konno, T. Yoshida, and F. Horikiri, Jpn. J. Appl. Phys. 56, o85503 (2017).

[26] D. F. Storm, M. T.Hardy, D. S.Katzer, N. Nepal, B. P. Downey, D. J. Meyer, T. O.McConkie, L. Zhou, and D. J. Smith, J. Cryst. Growth 456, 121 (2016).

[27] J. K. Hite, T. J. Anderson, L. E. Luna, J. C. Gallagher, M. A. Mastro, J. A. Freitas, and C. R. Eddy, J. Cryst. Growth 498, 352 (2018).

[28] K. Yoshida, S. Yamanobe, K. Konishi, S. Takashima, M. Edo, B. Monemar, and Y. Kumagai, Jpn. J. Appl. Phys. 58, SCCD17 (2019).

[29] H. Fujikura and T. Konno, Appl. Phys. Lett. 113, 152101 (2018). 
[30] F. Oehler, T. Zhu, S. Rhode, M. J. Kappers, C. J. Humphreys, and R. A. Oliver, J. Cryst. Growth 383, 12 (2013).

[31] A. Tanaka, K. Nagamatsu, S. Usami, M. Kushimoto, M. Deki, S. Nitta, Y. Honda, M. Bockowski, and H. Amano, AIP Adv. 9, 095002 (2019).

[32] I. C. Kizilyalli, P. Bui-Quanga, D. Disney, H. Bhatia, and O. Aktas, Microelectron. Reliab. 55, 1654 (2015).

[33] J. K. Hite, T. J. Anderson, M. A. Mastro, L. E. Luna, J. C. Gallagher, R. L. Myers-Ward, K. D. Hobart, and C. R. Eddy, ECS J. Solid State Sci. Technol. 6, S3103 (2017).

[34] K. Hayashi, H. Ohta, F. Horikiri, Y. Narita, T. Yoshida, and T. Mishima, Phys. Status Solidi A 215, 1700501 (2018).

[35] I. V. Markov, Crystal Growth for beginners; fundamentals of nucleation, crystal growth and epitaxy, (World Scientific PublishingCo.Pte. Ltd., 1941).

[36] P. Asbeck, III-Nitride Electronic Devices. Chapter One - Electronic properties of III-nitride materials and basics of III-nitride FETs, (Elsevier, 2019).

[37] H. Achour, S. Louhibi-Fasla, and F. Mana, Physics Procedia 55, 17(2014).

[38] S. Duman, S. Bağcl, H. Tütüncü, G. Uğur, and G. Srivastava, Diamond and Related Materials 15, 1175(2006).

[39] Y. Umeno, A. Kubo, and S. Nagao, Computational Materials Science 109, 105(2015).

[40] J. S. Galsin, in Solid State Physics, edited by J. S. Galsin (Academic Press, 2019), pp. $1-36$.

[41] M. Frentrup, L. Y. Lee, S.-L. Sahonta, M. J. Kappers, F. Massabuau, P. Gupta, R. A. Oliver, C. J. Humphreys, and D. J. Wallis, Journal of Physics D: Applied Physics 50, 433002 (2017).

[42] H. Zhong, C. Zhang, W. Song, K. Chen, Y. Sheng, G. Xu, and Z. Liu, Applied Surface Science 511, 145524(2020).

[43] S. Suandon, S. Sanorpim, K. Yoodee, and K. Onabe, Thin Solid Films 515, 4393(2007).

[44] H. Morkoc, Hanbook of Nitride Semiconductors and Devices, Vol. 1 - Materials Properties, Physics and Growth, (Wiley-VCH, 2008). 
[45] O. Ambacher, B. Foutz, J. Smart, J. R. Shealy, N. G. Weimann, K. Chu, M. Murphy, A. J. Sierakowski, W. J. Schaff, L. F. Eastman, R. Dimitrov, A. Mitchell, and M. Stutzmann, Journal of Applied Physics 87, 334(2000).

[46] C. E. Dreyer, A. Janotti, and C. G. Van de Walle, Phys. Rev. B 89, 081305 (2014, doi = 10.1103/PhysRevB.89.081305,).

[47] S. Mita, R. Collazo, A. Rice, J. Tweedie, J. Xie, R. Dalmau, and Z. Sitar, physica status solidi c 8, 2078(2011).

[48] T. Paskova, R. Kroeger, D. Hommel, P. P. Paskov, B. Monemar, E. Preble, A. Hanser, N. M. Williams, and M. Tutor, physica status solidi c 4, 2536(2007).

[49] V. Darakchieva, T. Paskova, M. Schubert, H. Arwin, P. P. Paskov, B. Monemar, D. Hommel, M. Heuken, J. Off, F. Scholz, B. A. Haskell, P. T. Fini, J. S. Speck, and S. Nakamura, Phys. Rev. B 75, 195217(2007).

[50] K. Lorenz, E. Wendler, A. Redondo-Cubero, N. Catarino, M.-P. Chauvat, S. Schwaiger, F. Scholz, E. Alves, and P. Ruterana, Acta Materialia 123, 177(2017).

[51] T. J. Flack, B. N. Pushpakaran, and S. B. Bayne, Journal of Electronic Materials 45, 2673(2016).

[52] F. Cimalla, J. Pezoldt, and O. Ambacher, Journal of Physics D: Applied Physics 40, S19(2007).

[53] V. Darakchieva, B. Monemar, and A. Usui, Applied Physics Letters 91, 031911(2007).

[54] D. Q. Tran, R. Delgado-Carrascon, J. F. Muth, T. Paskova, M. Nawaz, V. Darakchieva, and P. P. Paskov, Applied Physics Letters 117, 252102(2020).

[55] H. Sakakima, A. Goryu, A. Kano, A. Hatano, K. Hirohata, and S. Izumi, Journal of Applied Physics 128, O25701(2020).

[56] R. Kucharski, T. Sochacki, B. Lucznik, and M. Bockowski, Chinese Physics B 24, o66105(2015).

[57] H. Yamane, D. Kinno, M. Shimada, T. Sekiguchi, and F. J. Disalvo, Journal of Materials Science 35, 801(2000).

[58] T. Hashimoto, F. Wu, J. S. Speck, and S. Nakamura, Nature Materials 6, 568(2000).

[59] R. Kucharski, T. Sochacki, B. Lucznik, and M. Bockowski, Journal of Applied Physics 128, 050902(2020). 
[6o] T. Paskova, E. Valcheva, P. Paskov, B. Monemar, A. Roskowski, R. Davis, B. Beaumont, and P. Gibart, Diamond and Related Materials 13, 1125(2004).

[61] S. Fujimoto, H. Itakura, T. Tanikawa, N. Okada, and K. Tadatomo, Japanese Journal of Applied Physics 58, SC1049(2019).

[62] C. Kittel, Introduction to Solid State Physics. Seventh Edition, (Wiley, 1996).

[63] B. Yacobi and D. Holt, Cathodoluminescence Microscopy of Inorganic Solids, (Springer, Boston, MA, 1990).

[64] S. Mita, R. Collazo, A. Rice, R. F. Dalmau, and Z. Sitar, Journal of Applied Physics 104, $013521(2008)$.

[65] P. Reddy, S. Washiyama, F. Kaess, R. Kirste, S. Mita, R. Collazo, and Z. Sitar, Journal of Applied Physics 122, 245702(2017).

[66] W. Burton, N. Cabrera, and F. Frank, Philos. Trans. R.Soc. London, Ser A 2 243, 14336-14344 (1954).

[67] V. L. Tassev and D. F. Bliss, Journal of Crystal Growth 310, 4209(2008).

[68] K. Nagamatsu, Y. Ando, T. Kono, H. Cheong, S. Nitta, Y. Honda, M. Pristovsek, and H. Amano, Journal of Crystal Growth 512, 78(2019).

[69] T. Einstein, in Handbook of Crystal Growth (Second Edition), edited by T. Nishinaga (Elsevier, Boston, 2015), pp. 215-264second edition, ed.

[70] V. Jindal and F. Shahedipour-Sandvik, Journal of Applied Physics 106, o83115(2009).

[71] M. Uwaha, Progress in Crystal Growth and Characterization of Materials 62, 58 (2016).

[72] Y. Shen, R. Chen, X. Yu, Q. Wang, K. L. Jungjohann, S. A. Dayeh, and T. Wu, Nano Letters 16, 4158(2016).

[73] Y. Seta, A.-M. Pradipto, T. Akiyama, K. Nakamura, and T. Ito, physica status solidi (b) 257, 1900523(2020).

[74] V. I. Tokar and H. Dreyssé, Phys. Rev. E 71, 031604(2005).

[75] J. Elsner, R. Jones, M. I. Heggie, P. K. Sitch, M. Haugk, T. Frauenheim, S. Öberg, and P. R. Briddon, Phys. Rev. B 58, 12571(1998). 
[76] J. Colvin, R. Ciechonski, F. Lenrick, O. Hultin, M. Khalilian, A. Mikkelsen, A. Gustafsson, L. Samuelson, R. Timm, and B. J. Ohlsson, Phys. Rev. Materials 3, 093604(2019).

[77] H. Manasevit, Journal of Crystal Growth 13-14, 306(1972).

[78] H. Amano, M. Kito, K. Hiramatsu, and I. Akasaki, Japanese Journal of Applied Physics 28, L2112(1989).

[79] S. Nakamura, T. Mukai, M. Senoh, and N. Iwasa, Japanese Journal of Applied Physics 31, L139(1992).

[8o] B. Mitchell, V. Dierolf, T. Gregorkiewicz, and Y. Fujiwara, Journal of Applied Physics 123, 160901(2018).

[81] Z. Cheng, F. Mu, L. Yates, T. Suga, and S. Graham, ACS Appl. Mater. Interfacess 12, $8376(2020)$.

[82] S. Yngman, F. Lenrick, Y.-P. Liu, Z. Ren, M. Khalilian, B. J. Ohlsson, D. Hessman, L. Samuelson, R. Timm, and A. Mikkelsen, Review of Scientific Instruments 9o, 103703(2019).

[83] S. G. B., Organometallic Vapor Phase Epitaxy: Theory and Practice, (Academic Press, 1999).

[84] J. A. C. and H. M. L., Chemical Vapor Deposition: Precursors, Processes and Apllications, (Royal Society of Chemistry, 2008).

[85] O. Kordina, C. Hallin, A. Henry, J. P. Bergman, I. Ivanov, A. Ellison, N. T. Son, and E. Janzén, Phys. Status Solidi B 202, 321 (1997).

[86] A. Kakanakova-Georgieva, U. Forsberg, I. G. Ivanov, and E. Janzén, J. Cryst. Growth 300, 100 (2007).

[87] J. T. Chen, I. Persson, D. Nilsson, C. W. Hsu, J. Palisaitis, U. Forsberg, P. O. A. Persson, and E. Janzén, Appl. Phys. Lett. 106, 251601 (2015).

[88] J. T. Chen, C. W. Hsu, U. Forsberg, and E. Janzén, J. Appl. Phys. 117, 085301 (2015).

[89] H. Zhang, P. P. Paskov, O. Kordina, J.-T. Chen, and V. Darakchieva, Physica B: Condensed Matter 580, 411819(2020).

[9o] E. Yakovlev, R. Talalaev, A. Segal, A. Lobanova, W. Lundin, E. Zavarin, M. Sinitsyn, A. Tsatsulnikov, and A. Nikolaev, Journal of Crystal Growth 310, 4862(2008).

[91] O. Danielsson, C. Hallin, and E. Janzén, Journal of Crystal Growth 252, 289(2003). 
[92] A. Kakanakova-Georgieva, R. Ciechonski, U. Forsberg, A. Lundskog, and E. Janzén, Crystal Growth \& Design 9, 880(2009).

[93] D. Koleske, A. Wickenden, R. Henry, and M. Twigg, Journal of Crystal Growth 242, 55(2002).

[94] C. G. Van de Walle and J. Neugebauer, Journal of Applied Physics 95, 3851(2004).

[95] J. L. Lyons, A. Janotti, and C. G. Van de Walle, Applied Physics Letters 97, 152108(2010).

[96] O. Olsvik, O. A. Rokstad, and A. Holmen, Chemical Engineering \& Technology 18, 349(1995).

[97] M. D. Brubaker, S. M. Duff, T. E. Harvey, P. T. Blanchard, A. Roshko, A. W. Sanders, N. A. Sanford, and K. A. Bertness, Crystal Growth \& Design 16(2016).

[98] S. Swann, Physics in Technology 19, 67(1988).

[99] E. C. Knox-Davies, J. M. Shannon, and S. R. P. Silva, Journal of Applied Physics 99, 073503(2006).

[10o] M. Junaid, C.-L. Hsiao, J. Palisaitis, J. Jensen, P. O. Persson, L. Hultman, and J. Birch, Applied Physics Letters 98, 141915(2011).

[101] F. J. Giessibl, Rev. Mod. Phys. 75, 949(2005).

[102] E. Meyer, Progress in Surface Science 41, 3(1992).

[103] B. Warren, X-Ray Diffraction, (Dover Publications, INC.,New York, 1969).

[104] V. Darakchieva, T. Paskova, P. P. Paskov, B. Monemar, N. Ashkenov, and M. Schubert, Journal of Applied Physics 97, 013517(2005).

[105] M. A. Moram and M. E. Vickers, Reports on Progress in Physics 72, 036502(2005).

[106] T. Metzger, R. Höpler, E. Born, O. Ambacher, M. Stutzmann, R. Stömmer, M. Schuster, H. Göbel, S. Christiansen, M. Albrecht, and H. P. Strunk, Philos. Mag. A 77, 1013 (1998).

[107] V. Srikant, J. S. Speck, and D. R. k. Clarke, Journal of Applied Physics 82, 4286(1997).

[108] K. Vernon-Parry, III-Vs Review 14, 40(2000).

[109] D. B. Williams and C. B. Carter, Transmission Electron Microscopy.A Textbook for Materials Science, (Springer, Boston, MA, 2009). 
[110] P. J. Goodhew, J. Humphreys, and R. Beanland, Electron Microscopy and Analysis, (CRC Press, 2000).

[111] P. Corfdir, J. Ristić, P. Lefebvre, T. Zhu, D. Martin, A. Dussaigne, J. D. Ganière, N. Grandjean, and B. Deveaud-Plédran, Applied Physics Letters 94, 201115(2009).

[112] E. Meissner, S. Schweigard, J. Friedrich, T. Paskova, K. Udwary, G. Leibiger, and F. Habel, Journal of Crystal Growth 340, 78(2012).

[113] S. J. Rosner, E. C. Carr, M. J. Ludowise, G. Girolami, and H. I. Erikson, Applied Physics Letters 70, 420(1997).

[114] V. Darakchieva, T. Paskova, P. P. Paskov, B. Monemar, N. Ashkenov, and M. Schubert, Phys. Status Solidi A 195, 516 (2003).

[115] T. Paskova, P. P. Paskov, V. Darakchieva, S. Tungasmita, J. Birch, and B. Monemar, Phys. Status Solidi A 183, 197 (2001).

[116] T. Paskova, P. P. Paskov, V. Darakchieva, E. M. Goldys, U. Södervall, E. Valcheva, B. Arnaudov, and B. Monemar, Phys. Status Solidi C o, 209 (2003).

[117] R. Khan, A. Arora, A. Jain, B. S. Yadav, J. Lohani, A. Goyal, K. Narang, G. Upadhyaya, V. K. Singh, S. K. Saini, R. Raman, M. V. G. Padmavati, R. Tyagi, R. K. Bag, and U. Riaz, Journal of Materials Science: Materials in Electronics 31, 14336-14344(2020).

[118] J. Priesol, A. Šatka, F. Uherek, D. Donoval, P. Shields, and D. Allsopp, Applied Surface Science 269, 155(2013).

[119] A. Lotnyk, D. Poppitz, U. Ross, J. Gerlach, F. Frost, S. Bernütz, E. Thelander, and B. Rauschenbach, Microelectronics Reliability 55, 2119(2015).

[120] H. Fujiwara, Spectroscopic Ellipsometry, (John Wiley \& Sons, New York, 2007).

[121] V. Darakchieva, Strain-related structural and vibrational properties of group-III nitride layers and superlattices, (Linköping University, 2004).

[122] M. Schubert, Phys. Rev. B 53, 4265 (1996).

[123] T. E. Jenkins, J. Phys. D: Appl. Phys. 32, R45 (1999).

[124] R. D. Carrascon, D. Q. Tran, P. Sukkaew, A. Mock, R. Ciechonski, J. Ohlsson, Y. Zhu, O. Hultin, B. Monemar, P. P. Paskov, L. Samuelson, and V. Darakchieva, Phys. Status Solidi B 257, 1900581 (2020). 
[125] N. A. K. Kaufmann, L. Lahourcade, B. Hourahine, D. Martin, and N. Grandjean, J. Cryst. Growth 433, 36 (2016). 



\section{Papers}

\subsection{Publications included in the thesis}

\section{Paper I}

Rosalia Delgado Carrascon, Dat Quoc Tran, Pitsiri Sukkaew, Alyssa Mock, Rafal Ciechonski, Jonas Ohlsson, Yadan Zhu, Olof Holtin, Bo Monemar, Plamen P. Paskov, Lars Samuelson and Vanya Darakchieva

"Optimization of GaN nanowires reformation process by metalorganic chemical vapor deposition for device quality GaN templates"

Phys. Status Solidi (b), 257, 1900581 (2020)

I performed the AFM and XRD measurements and data analysis. I have done the calculations of defect densities and I have written the paper.

\section{Paper II}

Rosalia Delgado Carrascon, Steffen Richter, Muhammad Nawaz, Plamen P. Paskow and Vanya Darakchieva

"Homoepitaxial growth of GaN by hot-wall MOCVD : Thermal stability and effect of $\mathrm{H}_{2}$ " In manuscript

I have planned, designed and performed all growth runs. I have measured XRD, AFM, SEM-CL and performed data analysis. I have written the manuscript with the help of my co-authors. 


\subsection{Publications not included in the thesis}

\section{Paper I}

Dat Q. Tran, Rosalia Delgado Carrascon, John F. Muth, Tania Paskova, Muhammad Nawaz, Vanya Darakchieva and Plamen P. Paskov

"Phonon-boundary scattering and thermal transport in $A l_{x} G a_{1-x} N$ : effect of layer thickness" Appl. Phys. Lett., 117, 252102 (2020)

I have planned and performed the growth of undoped and Si-doped GaN epitaxial layers. 


\section{Papers}

The papers associated with this thesis have been removed for copyright reasons. For more details about these see:

https://doi.org/10.3384/9789179292478 


\section{FACULTY OF SCIENCE AND ENGINEERING}

Linköping Studies in Science and Technology, Licentiate Thesis No. 1928 (2022)

Department of Physics, Chemistry and Biology (IFM)

Linköping University

SE-58183 Linköping, Sweden

wWw.liu.se 